\title{
Boundary lubrication by adsorption film
}

\author{
Jun ZHANG, Yonggang MENG* \\ State Key Laboratory of Tribology, Tsinghua University, Beijing 100084, China \\ Received: 20 March 2015 / Revised: 21 April 2015 / Accepted: 20 May 2015 \\ (C) The author(s) 2015. This article is published with open access at Springerlink.com
}

\begin{abstract}
A complete understanding of the mechanism of boundary lubrication is a goal that scientists have been striving to achieve over the past century. Although this complicated process has been far from fully revealed, a general picture and its influencing factors have been elucidated, not only at the macroscopic scale but also at the nanoscale, which is sufficiently clear to provide effective instructions for a lubrication design in engineering and even to efficiently control the boundary lubrication properties. Herein, we provide a review on the main advances, especially the breakthroughs in uncovering the mysterious but useful process of boundary lubrication by adsorption film. Despite the existence of an enormous amount of knowledge, albeit unsystematic, acquired in this area, in the present review, an effort was made to clarify the mainline of leading perspectives and methodologies in revealing the fundamental problems inherent to boundary lubrication. The main content of this review includes the formation of boundary film, the effects of boundary film on the adhesion and friction of rough surfaces, the behavior of adsorption film in boundary lubrication, boundary lubrication at the nanoscale, and the active control of boundary lubrication, generally sequenced based on the real history of our understanding of this process over the past century, incorporated by related modern concepts and prospects.
\end{abstract}

Keywords: boundary lubrication; adsorption film; adhesion; surfactant; nanotribology; active friction control

\section{Introduction}

\subsection{Definition of boundary lubrication}

A comprehensive and popular definition of lubrication given by Wikipedia is as follows. Lubrication is the process or technique employed to reduce friction between, and wear of one or both, surfaces in close proximity and moving relative to each other, by interposing a substance called a lubricant between them. The lubricant can be a solid, a solid-liquid dispersion, a liquid, a liquid-liquid dispersion or a gas. As pointed out by Dorinson and Ludema in their textbook [1], such a dictionary definition of lubrication as the mitigation of friction and wear should be regarded as a description rather than a definition, as it does not answer what lubrication is, without understanding what friction and wear are, which are

*Corresponding author: Yonggang MENG.

E-mail: mengyg@tsinghua.edu.cn also two terms of phenomenological description and lack of rigorous scientific definition. In addition, the effects of a lubricant film are not limited solely to friction and wear, but also encompass other aspects of an interface, such as the surface energy, surface forces, electronic and ionic emissions, electric double layer, adhesion, and cohesion, which are of great importance in practice.

Although there are no well-accepted rigorous scientific definitions available for the term "lubrication", it has been well explored and clarified that there are two distinctive basic lubrication regimes, fluid film lubrication and boundary film lubrication (or boundary lubrication for short), in the accepted engineering terminology. Fluid film lubrication is the lubrication regime in which there is a continuous fluid film separating solid surfaces [2]. A characteristic of fluid film lubrication is that the external load on the solid surfaces is fully supported by the pressure generated within the fluid film. When a fluid film is created and 
maintained by an external pump forcing the lubricant within the space or gap between the parts, it operates in the hydrostatic mode. When the fluid film is formed and maintained by the viscous drag of the moving surfaces themselves as they are sliding relative to one another with a wedge, we say that it operates in the self-acting hydrodynamic mode. If the pressure in the fluid film is so high that deformation of the solids under the pressure is not negligible, and/or the changes in the viscosity and density of the interposing lubricant within the gap are no longer ignorable, such as under counterformal conditions, we call such a state elastohydrodynamic lubrication (or plastohydrodynamic lubrication when the deformation is plastic). The principle of fluid film lubrication is solely dominated by fluid mechanics and solid mechanics. The thermal effects on fluid film lubrication can also be taken into account.

Differing from fluid film lubrication, the lubricant under boundary lubrication does not possess fluidity regardless of where it originates from or whether it is generated by a gas, a solution, or other medium, and thus fluid mechanics fails to adequately describe the behavior of boundary films. Another characteristic of boundary film is that it covers the solid substrate in the form of molecularly thin layers, from a monolayer to multiple layers. In boundary lubrication, if the solid substrate is atomically smooth and/or the local pressure is insufficiently high to break down the intervening boundary film, the load is fully carried by the molecularly thin layer, as illustrated in Fig. 1. Usually, the external load is jointly borne by the boundary film itself and the distributed contacting asperities that penetrate off the thin boundary film for most rough engineering surfaces, as shown in Fig. 2. Upon the relative sliding of one rough surface against the other, a series of events rich in variation occur, including stick-slip motion, temperature rise, wear debris generation, material transfer, and tribofilm formation.

In the majority of engineering applications, a transition regime between the fluid film and boundary film lubrication regimes is often encountered when the operation conditions are changeable. The transition regime is called mixed lubrication. To deal with mixed lubrication, theories for both fluid film and boundary film lubrication are needed.

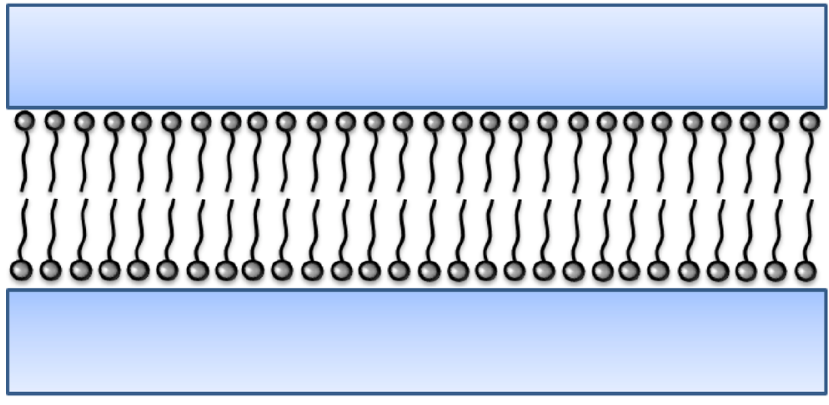

Fig. 1 Schematic diagram of Hardy's boundary lubrication model.

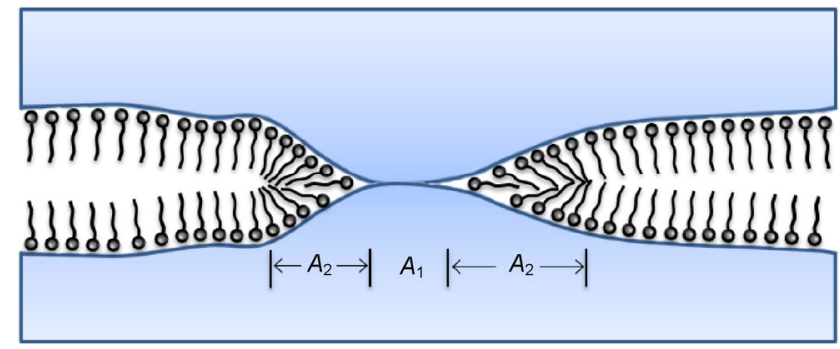

Fig. 2 Schematic diagram of Bowden's boundary lubrication model.

\subsection{The significance of boundary lubrication}

The effectiveness of boundary lubrication for the reduction in friction and wear can be demonstrated through a recreation of Leonardo da Vinci's friction experiments, which was recently reported [3]. The authors attempted to construct a nearly faithful recreation of Leonardo da Vinci's apparatus for measuring friction based on his notebook illustrations, and investigated the conditions under which Leonardo da Vinci conducted his experiments. They indeed reproduced Leonardo da Vinci's findings of static friction coefficients with wood of $\mu_{\mathrm{s}}=0.25 \pm 0.03$, but only when sliding blocks were used with roughly cut surfaces sullied by natural oils in fingertips and hands and by dust in the air. The static friction coefficient, $\mu_{\mathrm{s}}$ measured under dry, clean, smooth, and sanded conditions is $0.72 \pm 0.04$. Eliminating sanding from the experimental procedure lowers the static friction coefficient $\left(\mu_{\mathrm{s}}=0.44 \pm 0.04\right)$, and introducing fine olive wood sawdust on the sliding surfaces achieves an average static friction coefficient of $\mu_{\mathrm{s}}=0.35 \pm 0.03$. This study illustrates how sensitive static friction is to changes in surface roughness and contamination, or in other words, to the state of boundary lubrication.

Static friction has an extremely important significance 
in machine design and operation. A high static friction means that an extra-high resistance needs to be overcome, in addition to the inertial force or torque of the driven parts when starting or re-starting a machine. This is an especially important issue in the design of heavy-duty mechanical systems, such as those used in mining and metallurgical industries, in which a soft-start technology is required. For micro mechanical electrosystems (MEMS), on the other hand, as only a very limited driving force is available, if the static friction is high, overcoming the frictional resistance is difficult, thus giving rise to a stiction failure, or causing a severe unacceptable deformation owing to the weak microstructure involved. Even if the frictional resistance can be overcome with an adequate capacity of the driving system, the difference in magnitude between the static friction and kinetic friction, the latter being generally lower than the former, usually causes a stick-slip motion at each start of the machine, which is harmful for the stability of the mechanical system, especially for ultra-precision instruments such as a gyroscope. To obtain a smooth and quiet start, viscous type (i.e., near zero friction at the initial speed with a gradual increase under moving speeds) rather than Coulomb type (a step change in friction with movement) of Stribeck relationship is desirable. The coverage, microstructure, and behavior of boundary film, as well as the shear strength of asperity contacts (see Fig. 2), are the key factors determining the static friction, which are discussed in Sections 3, 4, and 5.

The significance of lubrication in reducing friction in industrial applications is often described by a plot of the so-called Stribeck curve (Fig. 3), which shows how the kinetic friction coefficient of a lubricated bearing changes with a lumped operation parameter, $B=\eta V / P$, where $\eta$ is the dynamic viscosity of the lubricant (Pa.s), $V$ represents the sliding speed $(\mathrm{m} / \mathrm{s})$, and $P$ denotes the specific load defined as $P=W / D L$ $(W$, external load $/ \mathrm{N} ; D$, bearing diameter $/ \mathrm{m} ; L$, bearing length $/ \mathrm{m}$ ). Parameter $B$, also regarded as bearing number, represents the intensity of operation condition for achieving full film lubrication. Only when $B$ is greater than a critical value can the lubricated bearing work in the full film lubrication regime, which is preferable to boundary lubrication or mixed lubrication because not only friction coefficient but also wear are

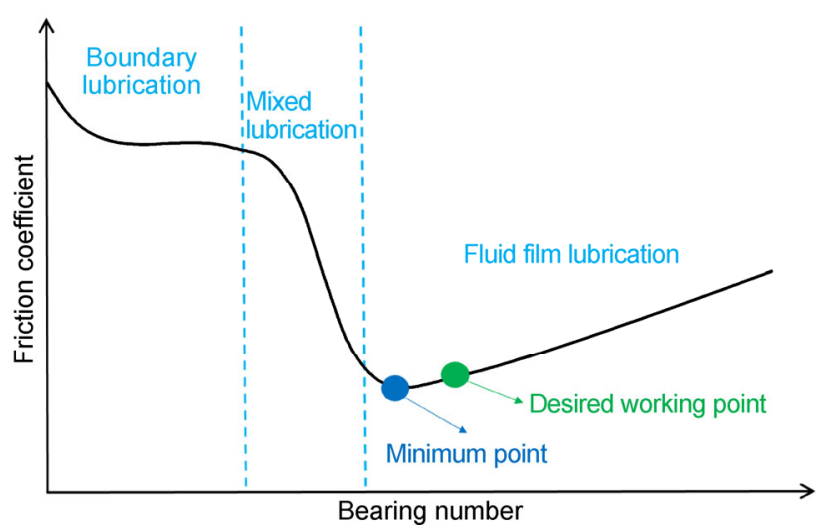

Fig. 3 Stribeck curve illustrating fluid film lubrication and boundary film lubrication regimes.

relatively low. The desired working point is in the vicinity of the minimum point of the curve. If the bearing number is in the right zone far beyond the desired working point, the viscous energy loss dissipated not only within the bearing but also in the entire lubricant circulation system will increase remarkably. Therefore, lower viscosity oils, such as $0 \mathrm{~W} / 20$ or $0 \mathrm{~W} / 10$, are better than $5 \mathrm{~W} / 40$ or $5 \mathrm{~W} / 30$ for engine lubrication, provided that full film lubrication of the engine parts will be maintained.

With the development of many smaller and heavyduty machine components, the specific load is continuously increasing, causing a continual reduction in the bearing number. Under the harsh conditions of elevated temperature, vacuum environment, solid lubrication, reciprocating movement around deadpositions, starved lubricant supply, and the starting and stopping processes, the contact surfaces may experience or even maintain at a working state at which the bearing number is close to zero, whereby boundary lubrication becomes dominant, and the friction coefficient therefore depends weakly on the bearing number, but strongly on the chemistry and microscopic structures of both the lubricant and the contact surfaces. In the boundary lubrication regime, the coefficient of friction usually increases and fluctuates much more than for a full film lubrication, and damage to the contact surfaces therefore becomes inevitable. This results in a worsening of the mechanical efficiency and the durability of the machine components. Unlike in full film lubrication, a quantitative prediction of the friction and wear during the design stage is very difficult for boundary 
lubrication, if not impossible, as there are no reliable theoretical models available. Therefore, most research studies on boundary lubrication have been conducted experimentally.

\subsection{Experimental techniques for characterizing boundary lubrication}

As described in Section 1.1 and illustrated in Fig. 2, a molecularly thin boundary film is sandwiched between two irregularly rough solids under a particular load. There are at least three significant obstacles for experimental exploration of boundary lubrication [4]. First, asperity contact occurs principally at a multitude of the summits of the surface roughness, meaning that the basic material volume affected by contact tends to be extremely small even at the nanoscale level, and is thus difficult to detect. Second, the heterogeneous mating interface is embedded deeply into two solids that are normally not transparent, and are thus inaccessible to most scientific characterization techniques. Finally, during the process of sliding, the contacting microstructures are not static, but evolve as the rubbing progresses.

To overcome these difficulties, various experimental techniques have been invented and developed, which are either used independently or along with traditional friction testers. Herein, we classify and briefly introduce the experimental techniques available for boundary lubrication investigations into three categories, precharacterization, in-situ characterization and postcharacterization, based on whether the experimental characterization is conducted before, during, or after the friction and lubrication tests.

Pre-characterization mainly focuses on one or both of the solid surfaces to be lubricated and contacted. The scope of the pre-characterization can cover the structural, physical, and chemical properties in a wide range from the macroscopic to atomic scales. There are a variety of scientific instruments and tools available for pre-characterization of a solid surface. Modern surface topography instruments, including various optical microscope, stylus profilometer, scanning tunneling microscope (STM), and atomic force microscope (AFM), enable researchers to quantitatively reveal $3 \mathrm{D}$ geometric surface structures down to the nanometer scale. The scanning electron microscope
(SEM) and tunneling electron microscope (TEM) have become powerful tools for high-resolution inspection and semi-quantitative characterization of surface and sub-surface structures. Hardness tester, nanoindentor and nanoscratching tester, contact angle meter, surface tension meter and various types of functional AFM are widely used to characterize the mechanical and other physical properties of a solid surface. To characterize the chemical composition and molecular structure of a solid surface, Auger electron spectroscopy (AES), electron spectroscopy for chemical analysis (ESCA), X-ray photoelectron spectroscopy (XPS), secondary ion mass spectrometry (SIMS), and Raman spectroscopy are also available. However, most electron, ion, or X-ray based tools require a high vacuum environment to accommodate the sample to be inspected, which means boundary films adsorbed on the solid surface are cleaned up. To measure the amount of mass and thickness of an adsorbed boundary film, quartz crystal microbalance (QCM) and ellipsometer are very useful tools.

An in-situ characterization of boundary lubrication and friction is much more difficult to achieve than pre-characterization because of the second and third obstacles mentioned above. AFM working in contact mode is often used to study boundary lubrication at the nanoscale, or at the microscale by replacing the tip with an attached microsphere, because of its measurement capability for normal and lateral forces. By equipping a miniature actuator and force sensor, SEM and TEM can be used to capture atomic images of the deformation and rupture of an asperity contact and the material transfer during the rubbing process. A limitation of such a powerful technique is that it is only possible to use under vacuum condition. In a vapor- or liquid-phase environment, which most boundary lubrication encounter, optical methods need to be used. Based on the high resolution of film thickness with the method of fringes of equal chromatic order (FECO), surface force apparatus (SFA) is a powerful instrument for the characterization of boundary film lubrication. Fourier transform infrared spectroscopy (FTIR), Raman spectroscopy, and sum frequency generation (SFG) are also used in studies on boundary lubrication, usually incorporated with a friction tester. A disadvantage of these optical techniques is the necessity to use an optically transparent 
contacting part. For non-transparent contacts, acoustic emission (AE) sensor and charge emission detector are useful tools for detecting basic physical events occurring during boundary lubrication.

All of the experimental techniques used for precharacterization of lubricated and rubbed surfaces are suitable for post-characterization. Although important, a correlation of the surface characteristics between pre- and post-characterizations in a point-to-point manner is difficult to achieve. In addition, nonintentional contamination and the adsorption/ desorption of the substances from/into the environment is a problem that needs to be prevented for precise post-characterization.

\subsection{Adsorption film and the structure of the present review}

According to the definition by IUPAC, adsorption refers to an increase in the concentration of a substance at the interface of a condensed and a liquid or gaseous layer owing to the operation of surface forces. The substance, regarded as adsorbate, is as general tiny particles of atoms, ions, or molecules from a gas, liquid, or dissolved-solid phase. The surface upon which the adsorbate resides once it is adsorbed is relatively much larger in size, and the body of the surface is regarded as adsorbent. The reverse process of adsorption is desorption.

Adsorption and desorption are surface-based processes driven by changes in the surface energy. They spontaneously occur, obeying the laws of thermodynamics. Therefore, they may appear anywhere and at any time in an intentional or non-intentional way, regardless of our awareness of them. Under certain conditions, the amount of adsorbate, or the adsorption film thickness, is determined by the equilibrium between the rates of adsorption and desorption, and the microstructure of the adsorption film is a result of the balance between the adsorbateadsorbate intermolecular energy, adsorbate-surface intramolecular energy, and the adsorbate-external field interactions.

When the adsorbate-surface intramolecular interaction mainly comes from the weak van der Waals forces, the adsorption is generally classified as physisorption. If the characteristic of the adsorbate-surface intramole- cular interaction is covalent bonding, the adsorption is classified as chemisorption. The formation of boundary films based on physisorption or chemisorption is described in Section 2 of this review. The effects of boundary film on the adhesion and friction of rough surfaces are detailed in Section 3, followed by a discussion in Section 4 of the behavior of the adsorption film in boundary lubrication. In Section 5, research progress on boundary lubrication by adsorption film at the nanoscale is highlighted. Finally, in Section 6, active control of boundary lubrication is introduced, and the role of electrostatic attractions and repulsions between charged adsorbates and a charged surface in the formation of boundary film are emphasized. Each section represents a focal point in revealing the fundamental mechanism of boundary lubrication from a certain era, generally sequenced based on the real history of our understanding of this process during the past century; the modern concepts of these topics are also naturally incorporated into each section.

Adsorption films are commonly boundary films in a gaseous or liquid phase environment. Under high vacuum conditions, however, adsorption film is absent or negligible, and thus solid film lubrication is the only available choice. Although solid film lubrication is an important branch of boundary lubrication, it is not included in this review owing to space limitations. Another important topic of boundary lubrication excluded from this review is chemical reaction film as there are already a number of review papers on this topic in the literature, see references [4-6] for examples.

\section{Formation of adsorption film in boundary lubrication}

\subsection{The function of adsorption film}

The first systematic study of boundary lubrication was conducted by Hardy [7] in the early 1920s, which was the start of intensive study on the mechanism of boundary lubrication for nearly a century. Hardy measured the static friction using a homologous series of paraffins, alcohols, and acids as lubricants. An amazing simple relationship was obtained that friction is a function of separate contributions by the solid 
surfaces, the chemical series to which the lubricant belongs, and the chain length of the lubricant molecules. To interpret these data, he created a picture illustrating the mechanism of boundary lubrication in which the molecules of the lubricant are oriented at each sliding surface to form a monolayer adsorption film (Fig. 1). The solids sink through the bulk lubricant until the surfaces are separated by only the unimolecular films of the lubricant adsorbed on each surface. As the lubricant molecules consist of a polar head group and an alkyl tail, the head groups adhere to the substrate when sliding, whereas the tails are exposed to be a closely packed layer, and the two boundary layers rub past each other instead of the surfaces themselves. Owing to the relatively weak shear strength of the van der Waals interactions between the adsorbed layers, lower friction can be achieved. Hardy also assumed that the extent of the reduction in the fields of surface force, which determines the adhesion force between the nonpolar tails of the adsorbed molecules, is a function of the molecule chain length, thereby explaining the linear relationship observed between the friction and molecular weight for different members of a homologous series.

Based on his experiments and analyses, Hardy emphasized the necessity for applying modern physical and chemical concepts and methods to the study of the lubrication process. The existence of orientated films was soon confirmed by X-ray scattering experiments [8]. Hardy's theory was also supported by Cameron [9], who used the Kirkwood-Müller formalism to calculate the van der Waals interactions, and thus deducing the friction force (1960). It is reasonable to suppose the interactions between the adsorbed molecules contribute to the total frictional force. However, as Hardy's experiments used an enormous amount of lubricant between the solid surfaces, the model he created was unable to be validated, and a question therefore remained regarding whether a monolayer of adsorbed film is able to provide boundary lubrication effect. This question was investigated by testing the lubrication properties of artificially prepared surface films, especially ordered molecular films such as Langmuir-Blodgett (LB) films and self-assembled monolayers (SAMs), which will be illustrated in the following sections. Another drawback of Hardy's experiments was that only static friction, and not kinetic friction, was recorded and considered in his paper, which was limited by poor instrumentation. Many complications can arise during motion owing to the complex and varying nature of the surfaces when in contact, such as fluctuations of the local pressure and temperature, which were later recognized to contribute to the rich phenomena in boundary lubrication.

\subsection{Langmuir-Blodgett films and their application in boundary lubrication}

LB films, named after the two scientists who invented this technique of film preparation, are single or multiple layers of amphiphilic molecules, deposited from the surface of a liquid onto a solid substrate. When a drop of dilute solution containing amphiphilic molecules with a hydrophilic head group and a hydrophobic tail is spread onto a water/air interface, the hydrophilic head group is preferentially immersed in water with the hydrophobic tail pointing toward the air. A monolayer of amphiphilic molecules with large spacing between the molecules (Fig. 4(a)) is then compressed by a barrier, forming a condensed and ordered molecular film (Fig. 4(b)). Finally, the monolayer on the water/air interface is transferred onto a solid substrate either by vertical deposition (Fig. 4(c)) or horizontal lifting method. Multilayer LB films can be

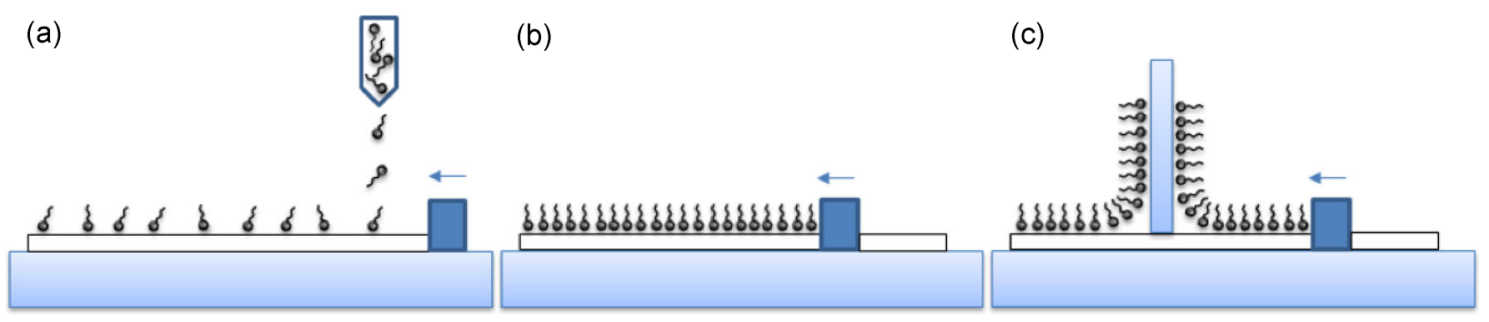

Fig. 4 Procedures for preparation of LB films: (a) spread amphiphilic molecules on water surface; (b) compress the molecules on water surface; (c) transfer the film onto a solid substrate. 
achieved by repeated deposition of single layers on a solid substrate, with the molecular direction changing alternatively for different monolayers (Y-type), or remaining in the same molecular direction $(X-$ or Z-type).

Using the newly developed LB technique by themselves, Langmuir [10] was the first to show that a single layer of fatty acid is sufficient to reduce the friction coefficient of glass surfaces from about 1 for clean glass to about 0.1 (1934). The main development in understanding the role of thin layers in boundary lubrication came from Bowden and Leben [11], who deposited specific layers of stearic acid on a steel plate surface using the same technique, and investigated the lubrication properties (1940). A steel slider was run across the coated steel plate repeatedly over the same track, and the friction during each run was observed. The process was continued for 100 runs, or until the surfaces were badly torn and the friction had increased to a significantly high level. The experiment was repeated using different numbers of films on top of the lower steel plate. A marked change in the behavior was observed during the running process, and the results obtained for various film thicknesses were recorded (Fig. 5). It is clear that the friction coefficient is almost as low as 0.1 even when a single layer of the film is present on the surface during the first run. Not only the friction coefficient but also the wear is quite similar to that found when excess stearic acid is present on the surface. However,

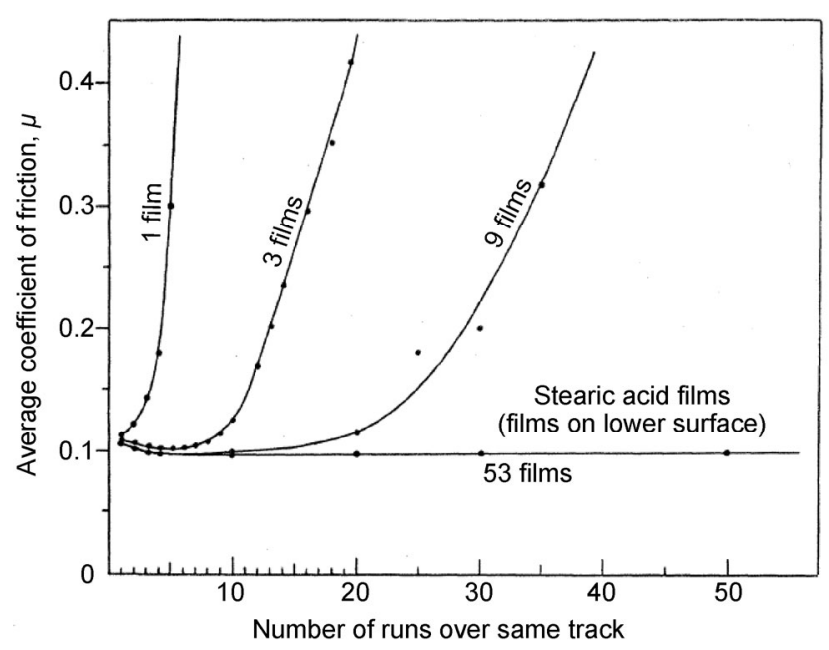

Fig. 5 Friction coefficient of steel surface coated by specific layers of LB films run over by slider on the same track [11]. when repeatedly running over the same track, the friction soon begins to rise, and eventually the friction and wear reach the same state as unlubricated surfaces. It is sufficient to conclude that the monolayer acting as boundary film is rapidly worn off as a result of the repeated sliding process. Similar results were obtained in the presence of other initial film thicknesses, but the rate of the boundary film being worn off was shown to decrease upon the increase in the thickness of the film. Eventually, when a sufficiently large number of film layers are present, the well-lubricated condition remains even after 100 runs along the same track. Bowden's experiments confirmed the importance that the thin adsorption layers of lubricant play in boundary lubrication. However, because of their complex preparation procedure and low resistance to sliding, LB films were gradually replaced by SAMs in studies on boundary lubrication.

\subsection{Self-assembled monolayers and their application in boundary lubrication}

Self-assembled monolayers are formed spontaneously through the immersion of an appropriate substrate into a solution of amphiphilic molecules. This technique is one of the most frequently studied systems at the molecular level because of its well-defined structure, strong head group binding on the substrate, and dense packing of the hydrocarbon chains [12], which are ideal for studies on boundary lubrication. Moreover, the way in which SAMs act as boundary lubrication film is very similar to that of additives functioning in commercial lubricating oils. Actually, chemical reaction films formed by some kinds of special additives such as ZDDP are in many circumstances more effective as boundary film [13], which have been reviewed in some high-quality papers [4-6], but not included in this article.

SAMs bind to a substrate surface by physisorption, chemisorption, or both. SAMs bounded by chemisorption usually consist of chain molecules with a head group that binds to a specific surface through chemical bonding, and a tail group that has desirable chemical qualities to the exposed interface, the binding of which is relatively strong, providing sufficient resistance to motion. The thiol group is a frequently used head group for attachment to a metal substrate such as 
gold or silver, and such alkanethiol SAMs are known to form highly ordered structures [14]. In general, a well-functional SAM through chemical bonding requires a special matching between the head group and the substrate, such as hydrolyzed trichlorosilane on silicon-based surfaces [15], and a carboxylic group on metal oxide.

Hydrophobic and electrostatic interactions are the dominant driving force for physically adsorbed SAMs formed from surfactant molecules, which are usually composed of a hydrophobic chain and a charged head group $[16,17]$. The aggregation structures of physically adsorbed SAMs are quite diverse depending on the properties of the surface, the molecular structure of the surfactant, and the concentration of the solution, in which monomers and spherical, cylindrical, and hemicylindrical aggregations are commonly formed [18]. Physically adsorbed SAMs are usually weakly attached, and are easily peeled off from the surface during the sliding process. Fortunately, a physically adsorbed film can recover very soon under a deficit, which may be the main reason for providing efficient boundary lubrication effect; for example, the SAMs formed by SDS in aqueous solution can recover very quickly, i.e., on the order of $10 \mathrm{~ms}$ on a graphite surface $[19,20]$. A physically adsorbed surfactant on a solid/liquid interface was reported to improve boundary lubrication properties significantly, and is especially efficient in water-based lubricants [21-24]. There are some other types of SAMs, such as polymer brushes, charged (polyelectrolyte) $[25,26]$ or neutral [27], adsorbed or grafted, on a solid surface [28], each of which has its own special tribological properties related to its unique structure. There was also an attempt to form an ionically bound polymer layer and mobile multilayer with self-healing effect to provide robust and long-lasting boundary lubrication performance [29].

In addition to friction and lubrication experiments, to elucidate the mechanism of boundary lubrication, a thorough inspection of the boundary film is necessary [24]. Adsorption isotherms have long been used to study the adsorption properties of SAMs on a solid/liquid interface [30], but it was far from satisfactory to investigate the adsorption at an interface at the molecular level. Actually, studies on the adsorption process of different interfaces have long been hampered by the need to discriminate between the few atoms at the interface, and the many more atoms that exist in the two bulk phases involved. A number of modern surface-sensitive techniques were developed in the late $20^{\text {th }}$ century to overcome this obstacle, such as AFM [31-33], electrochemistry [34], QCM [35-37], dual polarization interferometry (DPI) [38], and neutron reflectivity [39-41], to provide more information about SAMs on the solid surface both in air and in a liquid. A significant amount of useful knowledge has been acquired from these useful tools applied to SAMs, such as the physical and chemical properties of the molecules, the film thickness, the adsorbed mass, the aggregation structure, and the interactions between the molecules and between the molecules and solid surface. To provide a detailed introduction to the principle of such detection methods, and to provide useful guidelines on the criteria required to select the most appropriate techniques for studying a specific system, a comprehensive review of these modern techniques was made by Zaera [42]. The boundary lubrication properties of SAMs in both macroscopic and nanoscale tribology experiments are illustrated in the following sections.

\section{Adhesion between lubricated surfaces}

Ever since Hardy's study was first released, the important role played by a thin layer of adsorbed lubricant acting as boundary film has been widely recognized. However, it did not take long for researchers to realize that the rich behaviors of boundary lubrication cannot be solely attributed to the adsorption film. In the 1940s, adhesion between surfaces was found to be inevitable during the sliding process under boundary lubrication conditions, which was of vital importance in understanding the rich phenomena in boundary lubrication, such as wear and stick-slip. It was nearly at this same time that the adhesion between dry surfaces in air was acknowledged to play a major part in explaining Amontons' laws of friction. The time consistency was not only because both these works were led by Bowden and Tabor, but also because the experiments that the friction laws were derived from were also under somewhat unintended 
boundary-lubrication conditions owing to a boundary layer generated by the oxidation of the surface or by contamination [43]. Since then, a framework for boundary lubrication research has been built in which the quality of the boundary film is evaluated based on its ability to reduce direct contact or adhesion between solid surfaces, which is still the most efficient logic in interpreting boundary lubrication from a macroscopic or engineering perspective.

\subsection{The relationship between adhesion and Amontons' laws of friction}

The phenomenon in which the frictional force is directly proportional to the applied load regardless of the area of the sliding surfaces, known as Amontons' laws of friction (1699), can be traced back to the work of Leonardo da Vinci (1452-1519), which has been verified by an enormous number of engineering applications and everyday experience [44], but the interpretation is much more complicated. As surface roughness is obvious on surface, early insight is mainly focused on the interlocking of the surface asperities. Based on his observations, Coulomb concluded that friction is mainly determined by the work of lifting the load over the summits of these asperities (1781).

A breakthrough was made by Bowden [43], who conducted experiments to measure the real contact area by measuring the electrical resistance across the surface of the metals in contact (1950). The results show that the real contact area may be unexpectedly small, perhaps less than $1 / 10,000^{\text {th }}$ of the apparent area of contact. It is also amazing that the real contact area is directly proportional to the applied load, nearly independent of the surface size, shape, and roughness. Although contact stress will cause an elastic deformation in the vicinity of the points of contact, the experiments indicate that the summits of irregularities upon which the bodies are supported flow plastically, and are crushed down until their cross-section is sufficient to support the applied load. Based on these experiments, it is reasonable to correlate friction with adhesion, since the real contact area between the solids is proportional to the applied load, independent of the actual size of the surfaces, and Amontons' laws of friction can also be understood in this way. Later, based on the measured surface roughness data, Greenwood and Williamson [45], and Whitehouse and Archard [46], proposed contact models to describe the processes of contact between rough surfaces during sliding, including the asperity height distributions, skewness, and waviness of the surfaces. The real contact area was proved to be rigorously proportional to the load for rough surfaces because of elastic deformation, regardless of whether plastic deformation has occurred. A simple and elegant explanation of Amontons' laws of friction was thus achieved, which is still the basic understanding of friction process. Friction in air is also under boundary lubrication conditions by a boundary layer caused by the oxidation of the surface or contamination [43], as the friction coefficient will be much higher when the surface films are fully driven off at the condition that the contact surface is in a high vacuum.

\subsection{Adhesion between lubricated surfaces}

Bowden also found that plastic deformation and adhesion will also occur in the boundary lubrication regime, instead of the picture proposed by Hardy in which the surfaces are fully separated by adsorption films, although the adsorption film do play a major role in reducing friction and wear in boundary lubrication. The adhesion between friction pairs under boundary lubrication conditions was confirmed by Bowden and Leben [11] who took photomicrographs of the track and compared them with the unlubricated cases. The results show that the track of a lubricated solid surface is similar to, but somewhat smaller than, that of an unlubricated surface.

Based on a systematic study of the friction and wear of paraffins, alcohols, and acids as lubricants not only in bulk liquid but also as adsorbed monolayers, Bowden and Tabor [11,47-49] created a widely accepted model for boundary lubrication (1940). When lubricated surfaces are placed in contact, a plastic flow of the solid will occur until the area is sufficiently large to support the applied load. However, the pressure distribution is not uniform over the contact region. At certain points, the pressure will be much higher than average, and at these points, a local breakdown of the lubricant film may occur (area $A_{1}$ in Fig. 2). The breakdown of lubricant film will lead 
to the formation of contact junction or adhesion between the sliding surfaces, the size of which will be much larger than the lubricant molecule. Thus, the resistance of the transverse motion is the sum of the force needed to break the junctions between surfaces and the shear resistance generated by the lubricant film itself during the sliding process, which can be expressed as

$$
F=A\left[\alpha s_{\mathrm{m}}+(1-\alpha) s\right]
$$

where $A$ is the area supporting the applied load (including $A_{1}$ and $A_{2}$ in Fig. 2), $\alpha$ is the fraction of the area over which a breakdown of the lubricant film has occurred, $\alpha=A_{1} /\left(A_{1}+A_{2}\right), s_{\mathrm{m}}$ is the shear strength of the contact junctions, and $s$ is the shear strength of the adsorbed boundary film. For well-lubricated surfaces, the area of direct contact $\left(A_{1}\right)$ is very small compared with the real contact area, while the shear strength of these junctions is much higher than lubricant film so that they are responsible for the major part of the resistance to motion. The role of the adsorbed boundary layer is to reduce the amount of real contact area or adhesion between the sliding surfaces by interposing an intermediate layer, and possesses a relatively low shear strength while sliding. A framework for understanding boundary lubrication was generated. An enormous number of studies regarding the efficiency of boundary film to avoid adhesion between solid surfaces and the behaviors of the molecules in the boundary layer have since been conducted based on this framework.

\subsection{The relationship between adhesion and stick-slip phenomenon}

Bowden observed that stick-slip may occur when the lubricating performance of the boundary film is relatively poor. The transition between stick-slip friction, which is harmful to the friction pair, and continuous sliding, which is desirable, can be controlled by several factors [49]. Under the same conditions, continuous sliding with a low friction coefficient is easier to achieve using stearic acid as lubricant, which can form metallic soap on a steel surface, than using physically adsorbed films such as hydrocarbons and alcohol. The chain length is also an important parameter in this transition. Taking steel surfaces lubricated by fatty acids as an example, stick-slip friction occurs when the chain length is short, and continuous sliding is achieved with an appreciable reduction in friction and wear when the chain reaches a certain length of 5 or 6 carbon atoms, which corresponds to a molecular weight of about 100 . Another influencing factor is temperature. A transition from continuous sliding to stick-slip friction upon an increase in temperature may occur when the heating is insufficient to cause appreciable oxidation of the lubricant, and is reversible upon cooling. For pure paraffins and straight-chain alcohols, the transition occurs at the bulk melting point of the compound, and the transition temperature is sharp and clearly defined. With fatty acids, the transition temperature depends on the load, velocity, and other experimental conditions, but is usually higher than the bulk melting point of a fatty acid. Bowden was aware that the stick-slip phenomenon is related to the intermittent clutching caused by adhesion and breaking away of the surfaces, but he did not provide a more detailed explanation.

The stick-slip phenomenon, which is a central topic in boundary lubrication, but has yet to be clearly illustrated, has aroused the interest of scientists in many other disciplines. Actually, research on the stickslip phenomenon, characterized by a non-uniform relative motion between rubbing surfaces, is of vital importance in understanding articular cartilage damage [50], vibrations in vehicle suspensions and brake systems [51], erratic motion in industrial machinery and tools [52], and even active geological faults during an earthquake [53,54]. Although stickslip may be welcome as a way of generating elegant sound by musical instrument such as a violin, more often the appearance of stick-slip is detrimental as damage and wear of the materials are often caused by stick-slip motion accompanied by a harsh noise. The stick-slip motion or friction-induced vibration in an engineering system can be described and predicted by dynamic friction modeling and simulations [55-57]. Taking advantage of the high calculating efficiency of modern computers, dynamic friction modeling can reproduce the dynamic motion of a sliding system very close to the experimental results, 
even the hysteretic effects of friction, and is sufficiently powerful to provide credible guidance on the design of an engineering system. However, a model regarding the dependence of friction coefficient on sliding conditions such as the relative velocity, internal variables, and relative acceleration has to be pre-established before the simulation, as more of a semi-empirical formula of friction, which ignores the nature of the friction process. Another important achievement in understanding the mechanism of stick-slip is the phase transition model [58] established by shearing a confined thin layer of lubricant film by SFA and related molecule dynamic simulations, which is illustrated in greater detail in Section 5.1. However, an experimental condition in which there is no breakdown of the boundary film with atomic smooth sliding surfaces under very low contact pressure cannot be applied to normal macroscopic friction process.

Using $\mathrm{ZrO}_{2}$ ball and stainless steel plate as friction pair, Zhang and Meng [24] conducted a systematic study on the boundary lubrication performance, especially the stick-slip phenomenon in SDS aqueous solutions (2014). The SAMs formed on the steel surface by SDS molecules acts as boundary layer to provide lubrication during the sliding contact. As the SDS concentration increases, the mass of the adsorbed SDS molecules increases monotonously, whereas the structure of the adsorbed layer changes from monomers to hemimicelles, as illustrated in Fig. 6. The stick-slip phenomenon was studied (Fig. 7) at four typical concentrations of $0.01,0.1,1$, and $10 \mathrm{mM}$, representing the adsorption state with different adsorbed masses and adsorption structures. At the SDS concentration of $0.01 \mathrm{mM}$, regular stick-slip spikes are present, and repeat periodically at a constant driving velocity characterized by the static friction coefficient $\left(\mu_{\mathrm{k}}\right)$ derived by the force needed to initiate sliding from rest, and the kinetic friction coefficient $\left(\mu_{\mathrm{s}}\right)$ derived by the force needed to maintain the sliding process. As the concentration increases to $0.1 \mathrm{mM}$, both static and kinetic friction coefficients decrease. As the concentration increases to $1 \mathrm{mM}$, the stick-slip disappears, shifting to continuous sliding with a low friction coefficient of about 0.1 . When the concentration reaches $10 \mathrm{mM}$, at which the adsorbed SDS molecules form hemimicelles on the surface of stainless steel, stick-slip appears again as the kinetic friction coefficient remains at a low level, but the static friction coefficient becomes quite large. When the driving velocity increases, the kinetic friction coefficient stays nearly steady at a low value about 0.1 , whereas the static friction coefficient decreases until a critical driving velocity is reached, at which point the stick-slip friction turns into continuous sliding $\left(\mu_{\mathrm{k}}=\mu_{\mathrm{s}}\right)$. The dependence of the static friction coefficient on driving velocity results from the fact that the static friction coefficient depends mainly on the adhesion, which increases over time.

The general process of stick-slip friction can be concluded from these characteristics of stick-slip phenomenon. During continuous sliding or the slip process when stick-slip occurs, the adhesion, or $\alpha$, is mainly determined by the adsorbed mass of SDS on steel surface, regardless of the adsorption structure, so that the kinetic friction coefficient decreases monotonously as SDS concentration increases, and remains stable at about 0.1 when SDS concentration is sufficiently high. While during the stick process in stick-slip friction, $\alpha$ is related to both the adsorbed mass and the adsorption structure. When SDS molecules are adsorbed as monomers, the static friction coefficient also decreases with SDS concentration. However, when the SDS concentration is $10 \mathrm{mM}$,
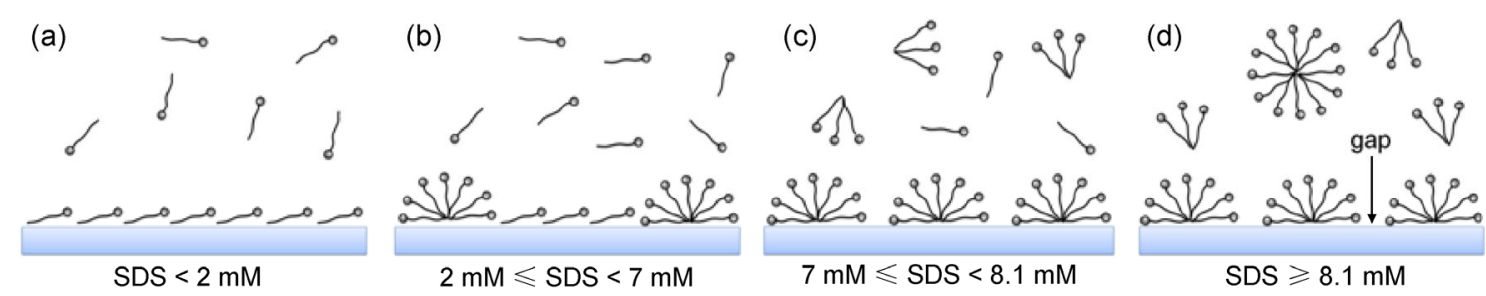

Fig. 6 Schematic diagram of four-stage adsorption model of SDS on 316L stainless steel surface in different concentrations; the sulfate groups and carbon chains of $\mathrm{DS}^{-}$are represented by the circles and curved lines, respectively [24]. 


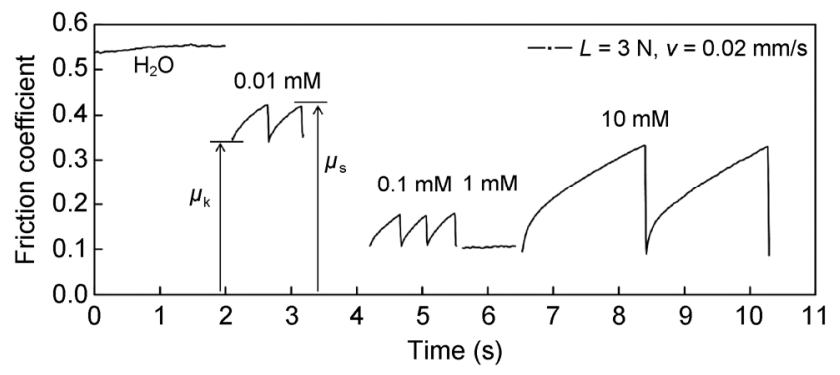

Fig. 7 Stick-slip phenomenon for different SDS concentrations at the load of $3 \mathrm{~N}$ and sliding velocity of $0.02 \mathrm{~mm} / \mathrm{s}$ [24]

although the adsorbed mass is high enough, the regular hemimicelles expose some area of bare surface, which facilitates the adhesion to grow, so that $\alpha$ is large and the static friction coefficient is high. The influence of sliding velocity on the static friction coefficient depends on the phenomenon that adhesion grows over time. When the driving velocity is low, there is sufficient time for the adhesion or $\alpha$ to grow during the stick process so that there is large stickslip spikes. As the driving velocity increases, the time for adhesion or $\alpha$ to grow becomes smaller and smaller until reaching the critical velocity, at which point stick-slip turns into continuous sliding. This research shows that stick-slip comes from the inconsistency of friction between sliding and sticking, and the boundary layer can affect them separately by influencing the adhesion between solid surfaces. The scenario of correlating the macroscopic boundary lubrication properties with the adsorption film behaviors at the nanoscale should be emphasized in the future.

\subsection{The effect of surface films on the plastic defor- mation of solid surfaces}

Material studies have shown that the presence of a surface film on solid surface can remarkably influence the mechanical behavior such as plastic deformation [59]. The effect of surface film on the plastic deformation (Fig. 8), and thus on the boundary lubrication properties (Fig. 9), was studied by Buckley [60] in 1972. Figure 8 shows a typical stress-strain curve for a material under a normal state, with surface-active liquid present on the surface (showing the Rehbinder effect), and with an oxide film present on the solid surface (showing the Roscoe effect) [61]. With the surface film present providing surface hardening or increased hardness with the Roscoe effect, the stress

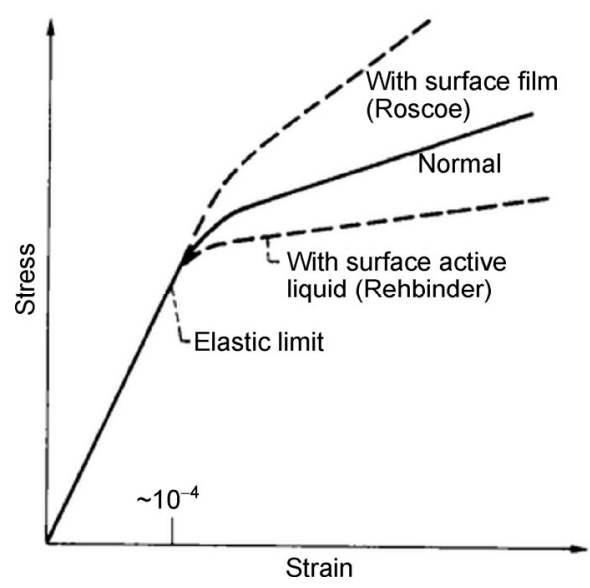

Fig. 8 Schematic illustration of principle extrinsic surface effects in the presence of surface films [61].
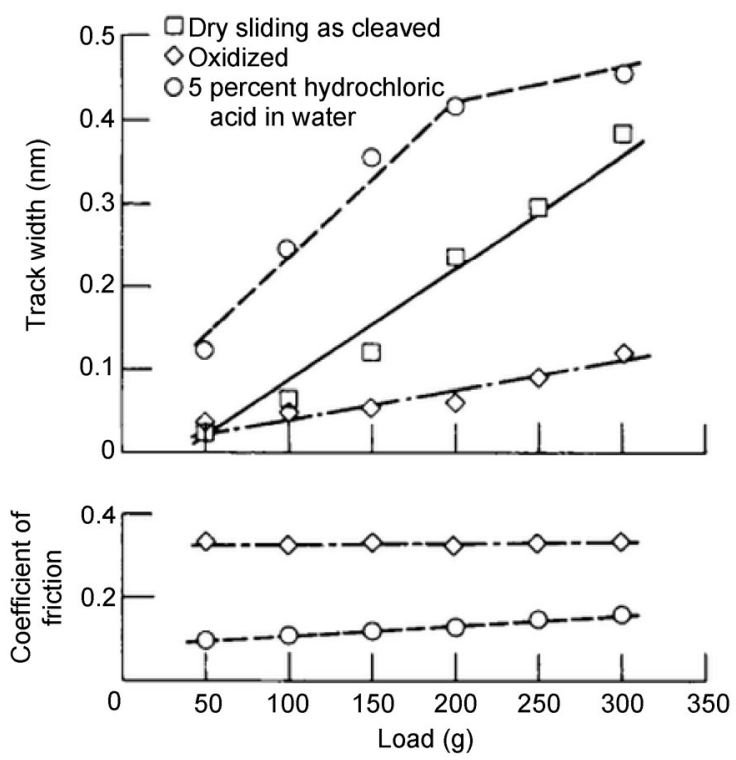

Fig. 9 Width of wear track and coefficient of friction achieved by ruby ball sliding on zinc single crystal (0001) surface in [101 0 ] direction with a sliding velocity of $1.4 \mathrm{~mm} / \mathrm{min}$ influenced by surface effects in the presence of surface films [61].

will increase for a given strain. With the application of a surface-active species to the solid surface, such as an organic acid which imparts a softening to the surface, the stress will decrease for a given strain because of Rehbinder effect. The curve of the normal surface lies between these two curves.

Both friction and wear characteristics are different under these three sets of surface conditions (Fig. 9). The oxidized zinc surface has the narrowest track width, indicating that plastic deformation is severely restricted by the oxide film. There is also a series of fine lines that look like lamellae running normal 
to the track, showing the restriction to plastic deformation that develops on the zinc surface during the deformation process, which is unique to the three conditions. On the contrary, the surface-active liquid increases the ductility or deformability of the solid surface, and thus the widest track is produced with pure plastic deformation. The track width data correlate completely with the stress strain data in that the track width decreases with an increase in the surface hardening. The friction coefficient is the lowest with the surface-active liquid during the sliding process, mainly because the shear resistance at the interface is lower with surface-active liquid than with surface oxide, which is not directly related to the mechanical properties of the substrate. Careful consideration should be taken regarding these surface effects in the presence of a certain type of surface film since plastic deformation needs to be strictly restricted in the friction pair of many machines. These surface effects will also contribute to the wear between lubricated surfaces, as described in the following section.

\subsection{Wear between lubricated surfaces}

It may be more important to reduce wear than friction in the boundary lubrication regime, as wear can cause the breakdown of the mechanical parts directly. Wear is also the core factor in understanding the running-in process, which is inevitable in contacting parts [62]. However, wear in boundary lubrication is a complex process, including both chemical attack and physical damage under many different conditions. Any slight change in the operation conditions may change the nature of the wear process [63]. Our understanding of wear process is much less than that of the friction process, and most studies on wear are largely empirically based.

When considering friction force in the boundary lubrication regime, it can be generally concluded that the breakdown ratio or adhesion strength between the surfaces will determine the friction force. However, when considering wear, the relationship between the adhesive junction and wear is much more complicated. When a junction is formed between the sliding surfaces, the shearing may occur in several different ways according to the strengths of the two substrates and the junction. If the junction is weaker than the two substrates, shearing will occur at the actual interface where the junction is formed, at which the wear will be very small although the friction may be high. If the weakest point exists in one of the substrates, shearing will often occur within the bulk of the weaker substrate, under which condition there will be a considerable removal of the softer material. If two similar substrates are used for boundary lubrication, the process of deformation and welding will work-harden them and appreciably increase the shear strength in the junction, so that the shearing will rarely occur at the interface but within the bulk of the substrates, which will lead to heavy surface damage on both sides.

Actually, any adhesion between surfaces will cause an increase in friction, but not necessarily an increase in wear. Based on previous experimental data on wear rate under different conditions, Archard [64] demonstrated theoretically that the wear rate is proportional to the load, and inversely proportional to the hardness of the substrate material, and the specific wear rate, $K$, is highly related to the probability of wear when an adhesive junction is formed (1953). Under boundary lubrication conditions, the boundary film will decrease the specific wear rate significantly. For metal surfaces under good boundary lubrication conditions, the value of $K$ is as low as $10^{-6}$ to $10^{-7}$, under which condition the adhesion between surfaces is appreciably reduced and most of the adhesion junctions do not produce wear. The lubricant films themselves also exhibit a marked difference in their resistance to wear [65]. For example, fatty acid films a few molecular layers thick are more resistant to wear than cholesterol film of the same thickness. Generally speaking, a film with a better capability of reducing friction is also more efficient at reducing wear. The surface mobility of the film and the ability to achieve a rapid adsorption from the bulk of the lubricant are also important factors in wear resistance.

Under different boundary lubrication conditions, the wear process may be influenced by many other factors beyond adhesion, such as chemical reactions, oxidation, corrosion, fatigue, and scuffing [66]. The specific wear rate, $K$, will change by several orders of magnitude according to these factors. In 1971, Beerbower [66] conducted a comprehensive survey of 


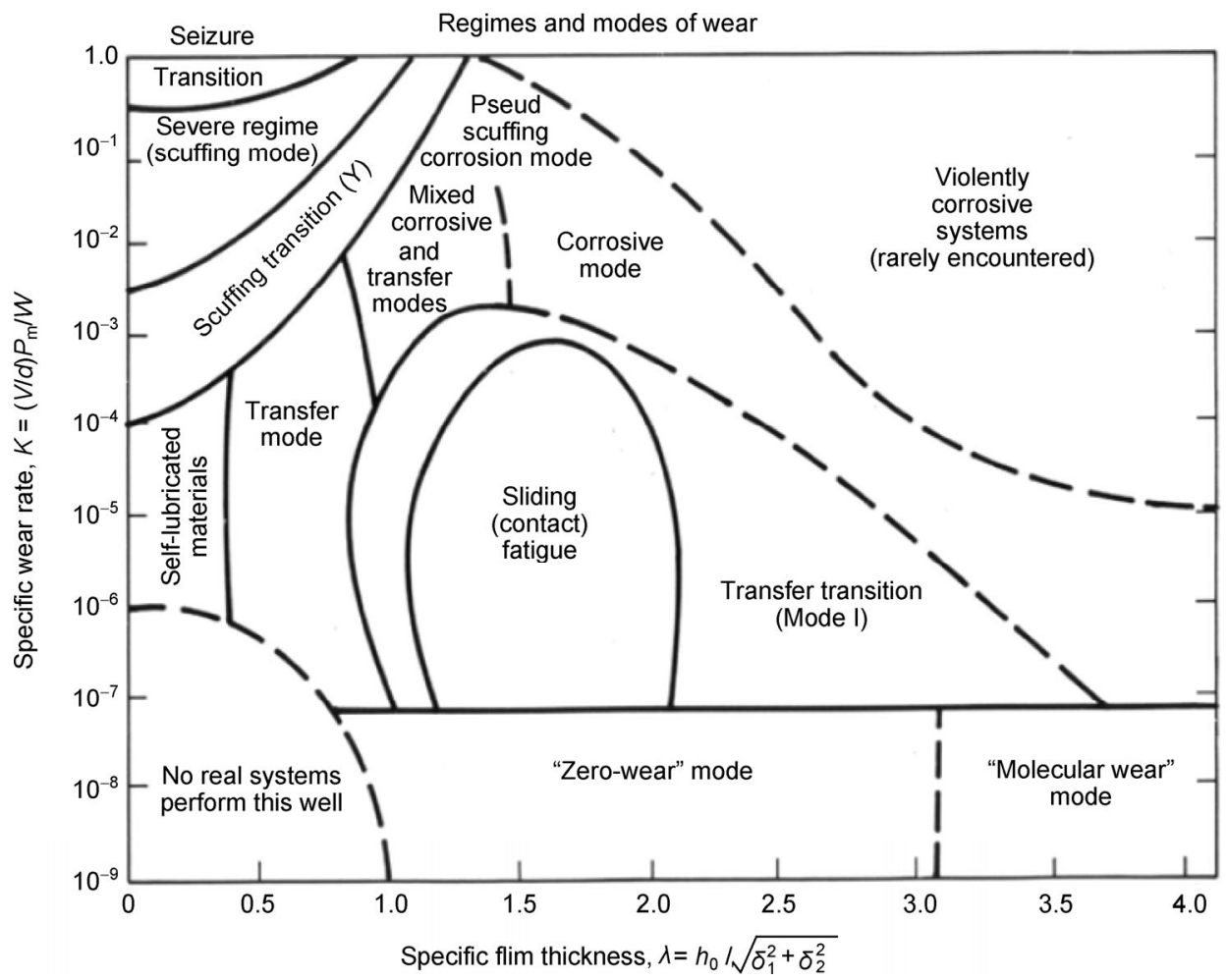

Fig. 10 Various wear mode in the boundary lubrication regime $\left(K, V, d, P_{\mathrm{m}}, W, \lambda, h_{0}, \delta_{1}\right.$, and $\delta_{2}$ are the specific wear rate, wear volume, sliding distance, hardness of material, external load, specific film thickness, minimum separation between the centerlines of the two roughness profiles, and roughness of the upper and lower surfaces, respectively ) [44].

the influencing factors, models, and mechanism of wear in the boundary lubrication regime, and drew a diagram illustrating the various modes of wear and lubrication mechanisms according to the specific film thickness and specific wear rate. This diagram, shown in Fig. 10, is based on a large number of experiments on the related conditions, and is very instructive for engineering and further studies of the wear mechanism.

\subsection{Summary of the adhesion between lubricated surfaces}

It is generally accepted that the mechanism of boundary lubrication between macroscopic sliding surfaces is the welding and shearing of rough opposing surfaces caused by a local breakdown of the boundary film, which has been the standard framework for the research on boundary lubrication. A number of complicated processes during boundary lubrication, such as stick-slip and wear, can be understood under this scenario. But there is no general agreement on how to predict the breakdown ratio $\alpha$ of boundary film precisely in a certain system since the value with large difference even not in the same order was reported in different systems [44]. Actually, the adhesion between surfaces is a dynamic process, as welding and shearing always occurs during the sliding process. Other factors such as the temperature and pressure distributions, as well as the behavior of the boundary film, should also be considered to achieve a more in-depth understanding of the boundary lubrication process.

\section{The behavior of adsorption film in boundary lubrication}

Owing to the persuasive studies conducted during the first half of the $20^{\text {th }}$ century, the important role of an adsorbed thin film as a lubricant in boundary lubrication became generally accepted, whereas the behavior of the boundary film itself in the sliding process was not well understood, and soon afterward became a focus of research. Methods based on mechanics, thermodynamics, and other factors were introduced in the description of the boundary film before a sufficient number of experimental techniques 
became available to directly probe the behaviors of boundary film molecules at the nanoscale.

\subsection{The dynamic properties of adsorption film}

Based on Bowden's adhesion model for boundary lubrication, Kingsbury [67] proposed a thermodynamic method (1958) to determine the breakdown ratio of boundary film $\alpha$, which was treated as related to the ratio of the time it takes for a single counterface asperity to pass the distance between two adsorbed molecules on the surface to the duration of the period when a molecule stays in the adsorbed state according to Frenkel's formula. This relationship can be expressed as

$$
\alpha=1-\exp \left[-\frac{Z}{v t_{0}} \exp \left(-\frac{E}{R T}\right)\right]
$$

where $\mathrm{Z}$ is the distance between the adsorbed molecules, $v$ is the velocity of the relative motion, $t_{0}$ is the period of vibration of the molecules on an order of about $10^{-14} \mathrm{~s}, E$ is the adsorption heat, $R$ is the gas constant, and $T$ is the absolute temperature. The friction coefficient in the boundary lubrication regime can be calculated by substituting $\alpha$ from Eq. (2) into Eq. (1) influenced mainly by $v$ and $T$, which are known to be important parameters in the friction process. Since Eq. (2) was derived based on the gas properties, which may not necessarily be accurately applied to liquid or solid molecules, the calculation of the friction coefficient on this basis is quite approximate. This model has also been adopted and modified, for example, by Rowe [68] for calculating the wear rate of rubbing bodies, and by Wang and Huang [69] for calculating the friction coefficient in boundary lubrication, testified experimentally somewhat in their sliding conditions.

\subsection{The behavior of adsorbed lubricant molecules under load}

The speculation regarding the pressure exerted on the lubricant film was made by Adamson [70], who was inspired by the discrepancy of Bowden's adhesion model in explaining the behavior of the electrical conductivity in boundary lubrication (1960). Under small loads, the conductivity is very small, as would be expected from Bowden's model, because of the small value of $A_{1}$ and the high electrical resistance of the film. However, under a higher load in which the lubrication performance is not diminished, the electrical conductivity increases to about the same as for unlubricated surfaces. From Adamson's viewpoint, the boundary film itself is under mechanical pressure, as the load is also supported by the boundary film. The pressure exerted on boundary film most probably comes about physically through deformation of the uneven substrate surface where the pressure is insufficient to form direct contact between surfaces by actually displacing the film, but is sufficient to place certain constriction, and hence pressure, upon it. As the real contact area is only a small fraction of the total area of apparent contact, only occasional small patches of the film undergo mechanical pressure. As a result, there is a tendency for pressurized film molecules to escape from the pressurized region to adjacent normal regions, as illustrated in Fig. 11. At the same time, the pressurized film may consist of molecules that are more or less lying flat. It is generally true that part of boundary film will be put under pressure, which brings about changes in the adsorption state of the film molecules during the sliding process, which should be considered and correlated to the boundary lubrication properties if possible.

\subsection{The behavior of lubricant molecules when sliding}

It can be dated back to the work of Hardy that the friction coefficient of boundary lubrication was found to be smaller with a larger molecular weight of lubricant molecules. An illustrative model called the cobblestone model was created to correlate this phenomenon with the behavior of lubricant molecules when sliding, which was first proposed by Tabor [71] (1981) and developed further by Homola [72], and has been efficient at explaining the interfacial friction at a low load with little or no wear. This model can be illustrated as pushing a cart over a road of cobblestones in which the wheels of the cart (which represent the

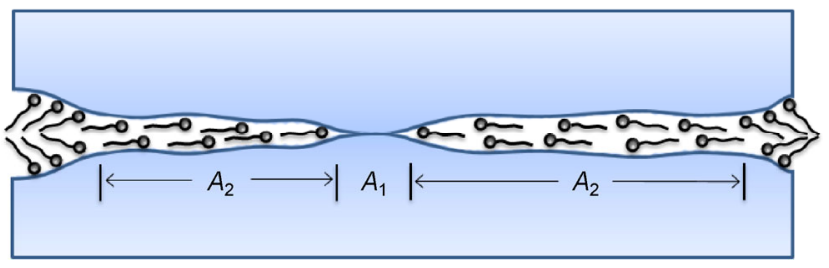

Fig. 11 Schematic diagram of Adamson's pressurized model for boundary lubrication. 
molecules of lubrication film) must be made to roll over the cobblestones (representing the molecules of the lower surface) before the cart can move. For the cart, the downward force of gravity represents the attractive intermolecular forces between the two material surfaces. When at rest, the wheels find grooves between the cobblestones where they sit in potential energy minima, and thus the cart is in stable mechanical equilibrium. A lateral force is required to raise the wheels against the gravity force to initiate motion. Pushing is needed for the motion to continue, during which energy is dissipated by the liberation of heat each time a wheel hits the next cobblestone. In this model, both the externally applied load and the attractive intermolecular forces are considered to have an effect on the boundary lubrication properties. It is intuitively clear that the larger the liquid molecules, the smoother the surfaces appear to the wheels, and hence the easier for them to roll over the surfaces. This is a good descriptive model for understanding the chainlength effect under certain boundary lubrication conditions, especially with spherical lubricant molecules. Furthermore, the modern concept of energy dissipation can also be incorporated in such a model [73].

\subsection{Summary of the behavior of adsorption film in boundary lubrication}

The above studies and speculations regarding the behavior of the thin-layer adsorbed lubricant molecules were endeavors aimed at an understanding of boundary lubrication at the molecule level. Although very crude with many unsatisfactory features, the attempts at interpreting boundary lubrication in different ways each fit certain aspects of the observed boundary lubrication properties, clearly show the complicated nature of boundary lubrication and the need for additional cross-disciplinary studies. These models also represent the transition from macroscopic view of boundary lubrication to the microscopic or nanoscale perspective, which became a focus of later scientists, as discussed in the following section.

\section{Boundary lubrication at the nanoscale}

The most significant development during the past several decades for boundary lubrication came from experiments conducted to acquire information regarding the lubrication properties at the molecular level. As illustrated in Section 3, at most interfaces of technological relevance, contact occurs at numerous asperities. Consequently, the importance of investigating single asperity contacts in studies on the fundamental tribological properties of an interface has long been recognized [74, 75]. The emergence and improvements in AFM and SFA have allowed systematic investigations into the interfacial problems with a sufficiently high resolution. Microscopic information on boundary lubrication is important not only from a fundamental perspective but also because of the increasing demand in understanding the lubrication behavior of ultra-thin lubricant films on sooth solid surfaces, especially in high-density magnetic recording technology and micro/nanoelectromechanical systems (MEMS/NEMS) [76].

\subsection{Experimental techniques in investigating boundary lubrication at the nanoscale}

Studies on boundary lubrication at the nanoscale are quite different from those on conventional tribology or macrotribology. Experiments are conducted with a relatively small mass under lightly loaded conditions. Under this situation, negligible wear occurs, and the surface properties dominate the boundary lubrication performance. Interfacial phenomena with atomic smooth surfaces involving ultra-thin films (as low as $1 \mathrm{~nm}$, which is close to the size of a lubricant molecule) are a main concern [77]. As the relatively sliding velocity between surfaces is very small, the sliding consistently falls into boundary lubrication state far from the hydrodynamic lubrication regime under lubricated conditions.

The utilization and skilled operation of special experimental equipment are of vital importance in acquiring accurate boundary lubrication information at the nanoscale. The AFM and SFA are the main facilities used in the study of boundary lubrication at the nanoscale. The AFM was first developed by Binnig et al. [78] in 1986 based on the design of the scanning tunneling microscope (STM) [79], and was used to measure ultra-small forces present between the AFM tip and the sample surface, the principle of which is shown in Fig. 12. Later, the AFM was modified to 
measure friction force as well, a version that is generally called friction force microscope (FFM) or lateral force microscope (LFM) [74, 80], which are used in studies on tribology at the nanoscale. The normal and lateral forces are usually measured by detecting the bending and torsional deflection of the cantilever supporting the tip. These two types of deformations can be recorded simultaneously by processing the signals in a four-quadrant photo detector irradiated by a laser beam reflected from the back of the cantilever. The spring constants of the cantilever and the sensitivity of the photo detector should be determined $a$ priori to derive the load and friction force from the measured deformations. The adhesion force can be also obtained by measuring the force-distance curve [81], where the cantilever bending is plotted versus sample displacement, and by recording the largest tensile load of the cantilever during the retraction [82].

The SFA, first developed in the late 1960s [83], is commonly employed to study both static and dynamic properties of molecularly thin liquid films sandwiched between smooth surfaces [84]. The SFA [85] consists of a pair of atomically smooth sheets (usually mica) mounted on cross-placed cylindrical lenses that can be pressed into contact, the principle of which is shown in Fig. 13. Actuators attached to the surface supports are used to exert normal or shear forces and to control the spacing between surfaces down to the angstrom level. The contact area and surface separation are usually measured by interference method with angstrom precision. Piezo bimorphs are attached to one of the surfaces to impart a lateral displacement or oscillation for friction and viscosity measurements.

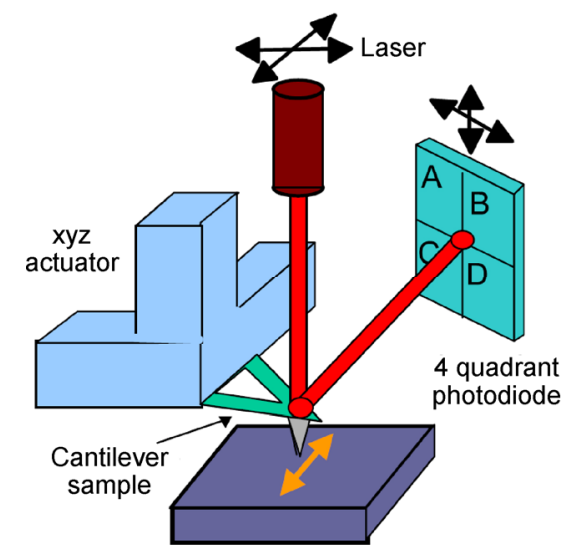

Fig. 12 Schematic diagram of the principle of AFM [82].

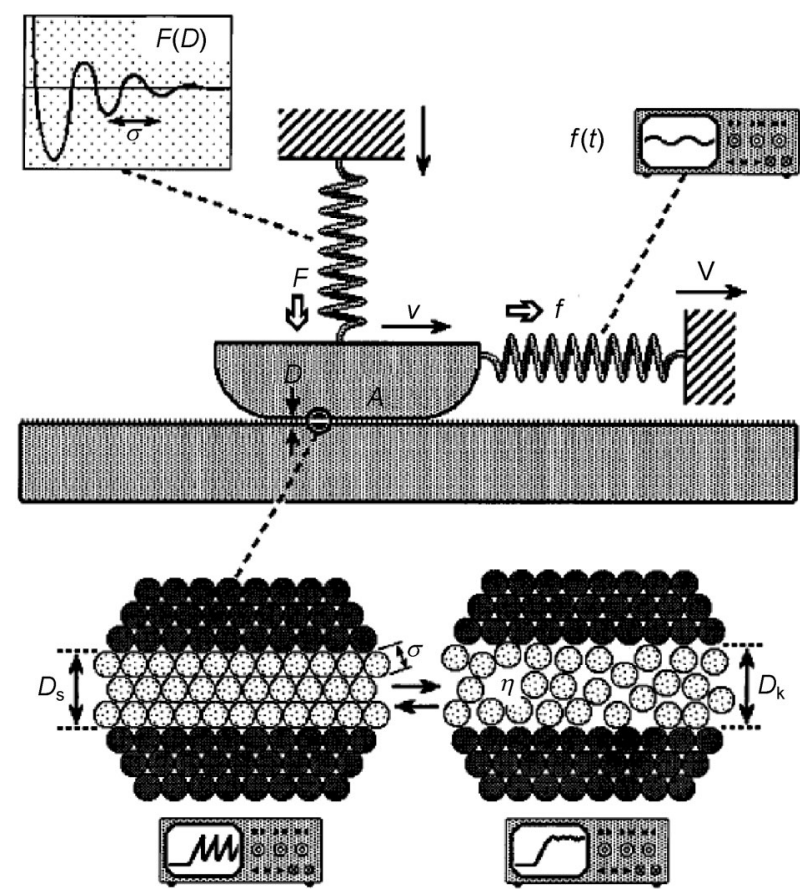

Fig. 13 Schematic diagram of the principle of surface force apparatus and the related phase transition model for boundary lubrication [77].

The atomic flatness of a surface is a necessity in achieving an atomic resolution. The materials usually used in studies on nanotribology include mica [86] and graphite [74], which have large areas with an atomically smooth surface that can be achieved by peeling as a result of the special layered structure of the materials, and silicon and silicon-based materials such as silica [15], which are acquired by polishing a silicon surface to an atomic smoothness and related post-treatment process. Gold, which is an inert metal, can be flattened, either by annealing [87] or sputtering/ evaporating [33], on an atomically smooth surface. Other metals such as steel [23] can be highly polished, and are thus candidates in these types of studies. Particularly, the material used in an SFA experiment should be flexible thin layer for mounting on the cylindrical lens, and should be optically transparent, so that mica is the best choice for such a material. On such atomically or relatively smooth surfaces with a roughness at the nanometer or sub-nanometer scale, the LB films and SAMs can be prepared in a more ordered and packed manner, which is ideal in studies on boundary lubrication at the nanoscale. 


\subsection{Phase transition of a confined thin layer of lubricant and related boundary lubrication properties}

When confined as a thin layer with a thickness close to a small multiple of the molecular diameter, the behavior of a liquid can no longer be described even qualitatively in terms of the bulk properties. With a high precision of the separation control of the SFA, the film will show a repulsive thinning with increasing pressure, where the liquid film is squeezed out of the contact area one molecular layer at a time. The layering transition was first observed by Gee et al. [88], and has since been explored extensively both experimentally [89] and theoretically [90] (1990). Furthermore, the effective viscosity of the thin film may be severalorders of magnitude larger than the bulk value, showing solid-like behaviors. If this occurs, the molecular configuration during the sliding process will be complicated when one of the surfaces is made to move laterally or to shear past the other. Under such conditions, the film alternately melts and freezes during the motion, resulting in stick-slip friction, as schematically shown in the lower part of Fig. 13. Stick occurs when the film is solid-like, giving rise to the static friction force, and slip occurs in the shearinduced molten state, giving rise to the kinetic friction force [58]. The stick-slip friction can be shifted to continuous sliding dependent on the sliding velocity, temperature, and load, which are factors influencing the behavior of the first-order transition between a solid- and liquid-like state of the film [91]. For example, there is a critical velocity above which the stick-slip will turn into continuous sliding. Actually, the phase transition is a much more complicated process, showing several types of ordered structures, in which the parameters deriving the dynamic transitions such as the velocity, temperature, and load are highly correlated with each other [58]; for more systematic information, refer to the work by Ruths and Israelachvili [92]. The phase transition model for boundary lubrication has been widely accepted for such a special system as proved by the enormous number of conducted experiments and computer simulation results [92].

Compared to the stick-slip behavior of a macroscopic friction system, the stick-slip originating from a thin layer of confined film shows certain similarities. For example, a transition from stick-slip friction regime to continuous sliding can be induced by an increase in velocity. However, the mechanisms between the two systems are completely different. For macroscopic friction conditions, the stick-slip phenomenon is mainly related to the breakdown ratio of the boundary film or the adhesion strength, whereas the occurrence of stick-slip during the sliding when lubricated by a thin film with a nanometer thickness is mainly caused by the solid-like to liquid-like transition of the film.

\subsection{Hydration lubrication}

Since the boundary lubrication properties of a confined thin layer of organic lubricant was intensively studied using SFA, a question was then raised regarding the behavior of a thin layer of water in such a system, which led to the theory of hydration lubrication. The nature of hydration lubrication is particularly relevant to the unique properties of water [93]. For most nonassociating liquids, such as organics and oils, the solid phase is denser than the liquid phase, so that the liquid has a tendency to become solid-like with a much higher viscosity, when confined between two surfaces sliding past each other across films that are only a few monolayers thick. In contrast, the liquid phase of water is uniquely denser than the solid phase, so that water retains a liquid-like fluidity even when confined between solid surfaces in films as low as a single layer in thickness, as the densification of the confined thin water film tends to suppress its tendency to solidify.

A hydration layer in which water molecules are tightly bound to ions or ionized surfaces in an aqueous medium because of their large dipoles can be viewed as a special form of an extremely thin water film. The hydration of charges leads to a strong repulsion when they approach each other to within a few nanometers or less, arising from the reluctance of the ions or surfaces to shed their hydration sheath. The shear properties of such a system were first studied by Raviv and Klein [94] in 2002, and a remarkably low friction coefficient of no greater than 0.0002 was achieved with a mean contact pressure of $0.3 \mathrm{MPa}$. During the sliding process, as the shear rate is much lower than the relaxation rate of the hydration shells, the hydrated 
ions will respond to shearing in a liquid-like fashion [95]. The ultra-low friction coefficient arises from the hydration lubrication mechanism in which the hydration layer sustains a high normal pressure and behaves as a fluid under shearing at the same time, as shown in Fig. 14. This type of hydration lubrication mechanism was later found to function in aqueous systems with charged ions, such as hydrated polymer brushes [96], amphiphilic surfactants [97], and liposomes [98]. The lubrication in biology was also expected to be somewhat related to the hydration lubrication mechanism [99], in addition to other frictional dissipation processes.

The hydration lubrication mechanism, research into which was led by Klein's group during the past decade, is a new perspective for understanding and controlling the boundary lubrication process in an aqueous medium. The critical point here is that the hydration shells surrounding charged, zwitterionic, or polar groups are tenaciously attached and are thus able to support large amounts of normal stress without being squeezed out; at the same time, they are also fluid so that the shear stresses can be very low when they slide past each other or past surfaces. The charisma of this system comes from the fact that an ultra-low friction coefficient can be achieved in such a simple system by a liquid found in everyday life. Problems remain as this theory is now deemed unable to systematically identify and rank charged groups, or their combinations, to determine their hydration lubrication efficiency [100]. When salt ions are combined with other lubrication additives, it is not easy to distinguish

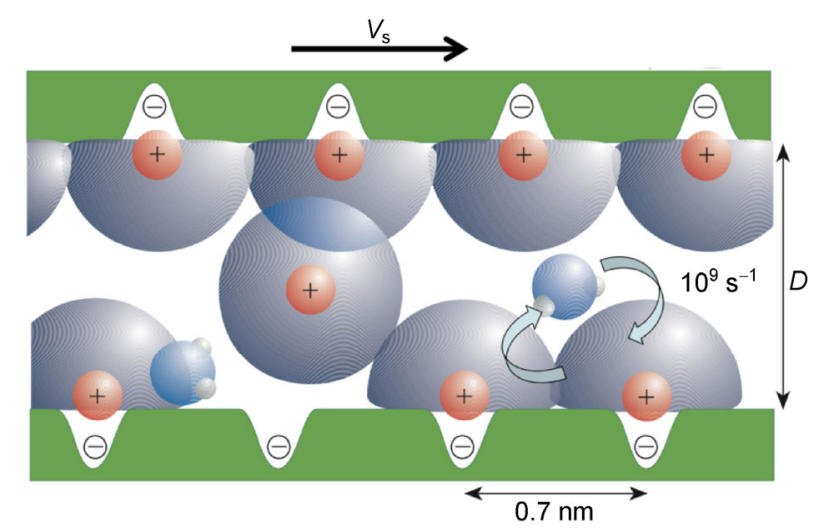

Fig. 14 Schematic diagram of the mechanism of hydration lubrication between charged surfaces across trapped hydrated ions [100]. whether the lubrication performance comes from hydration lubrication or another mechanism such as the influence of salt ions on the adsorption state [101] or conformation [102] of organic adsorption film. Another challenge comes from whether and how such a system can be applied to normal macroscopic boundary lubrication experiments or even to real machines as a way to dramatically reduce the amount of energy dissipation.

\subsection{Boundary lubrication in single asperity contact at the nanoscale}

Using AFM and the improved version of LFM, the boundary lubrication properties in a single asperity contact at the nanoscale can be investigated between a nanoscale tip and a smooth surface coated by SAMs. For well-bonded SAMs on a substrate, the friction process is just like a tip sliding on the top of molecular springs or a brush [103], which has compliant features and can experience an orientation and compression under a particular load. The friction and wear of such a process is usually very low until reaching a critical load at which the SAMs are worn away by the tip (Fig. 15).

Interesting results have been achieved regarding the effect of chain length on friction, which is one of the most fundamental questions regarding the nanotribological properties of alkylsilane SAMs. Salmeron's

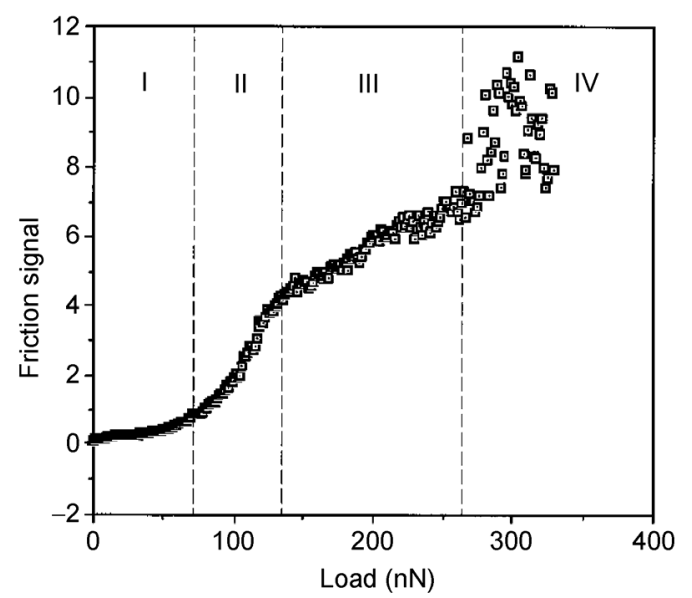

Fig. 15 Dependence of friction on load for a C18 alkylsilane monolayer on mica, in which four distinct regime exist: ( I ) elastic regime; ( II ) distortion and displacement of the SAMs; (III) tip in contact with mica substrate; (IV) wear of mica substrate [104]. 
group [104] conducted early studies on the chain length effect using alkylsilane chains of different lengths on mica probed by a $\mathrm{Si}_{3} \mathrm{~N}_{4}$ tip (1996). The general results show that the friction decreases with an increase in the chain length. As the load increases beyond $300 \mathrm{nN}$, the plot of the friction response shows distinct regimes corresponding to the elastic response of the coating, plastic deformation and displacement of the coating, contact of the tip with the substrate, and substrate damage, as shown in Fig. 15. The main reason for this was concluded that the increasing order being stabilized by the van der Waals attractions as the increase in the chain length promotes a reduction in friction. Additional studies were carried out regarding the chain length effect on friction, in which similar $[105,106]$ or different [107] results were reported. Although the chain-length effect on the friction at both the macroscopic scale and the nanoscale shows a similar trend, their mechanisms are different, as molecular dynamics simulation has shown that the poor lubrication performance of shorter chain alkanes at the macroscopic scale may lie in their inability to effectively separate the asperity contact between the sliding surfaces [108].

Some other factors of SAMs were investigated regarding their boundary lubrication properties. Loosely packed and disordered SAMs were shown to exhibit higher interfacial friction than those that were well packed and highly ordered, which is attributed to the stronger interaction with the tip by an enhanced area of contact, and the increased van der Waals interactions of liquid-like SAMs as compared with crystalline SAMs [14]. The terminal functionality was found to influence the friction at the nanoscale. For example, the nanoscale friction of the SAMs was measured to be on the order of $-\mathrm{COOH}>-\mathrm{OH}>-\mathrm{CH}_{3}$ for the same chain length [12]. The higher friction for - $\mathrm{COOH}$ functionality at the nanoscale was explained by the formation of hydrogen bonds between the contacting functional groups. It was also reported that mixed SMAs composed of two molecules with different chain lengths may have much lower friction than pure SAMs, which is caused by the structure of a highly ordered under-layer and a disordered, mobile outer-layer/canopy of the mixed SAMs [109]. A question quickly arises regarding how these factors influence their macroscopic boundary lubrication properties when the scale is changed by several orders of magnitude.

\subsection{The application of boundary lubrication at the nanoscale}

Generally speaking, studies on boundary lubrication at the nanoscale is far from the working conditions of machines in the boundary lubrication regime. However, there are some areas of industry in which the SAMs at the nanoscale can act as an efficient boundary film. The first is MEMS devices, which are utilized in a large number of successful products such as accelerometers, gyroscopes, and pressure sensors. The study of the nanotribology of SAMs is in fact closely related to the development of MEMS, where the high surface-to-volume ratio makes the interfacial interactions a main factor in the wear and lifetime of such devices [110,111]. The materials used in MEMS are mainly silicon based, the fabrication process of which was developed from the microelectronics industry; thus, mass production can be achieved. The release process to free the movable parts will always lead to an undesirable adhesion, the effect of which is generally referred to as stiction. SAMs are ideal in reducing the amount of stiction as the head groups can be chosen to attach to an oxidized silicon substrate, whereas the hydrophobic tail groups can greatly reduce the adhesion and interfacial friction. SAMs have been used to control stiction and friction in the contacting parts to achieve a better operational performance [112]. The ability of SAMs to withstand processing environments such as high temperatures and mechanical stresses from contact, and the packing densities, are also important concerns in the design of SAMs used in MEMS products [76].

Another successful example is the hard disk drive, which has become a necessary device in our daily lives. A disk drive consists of a magnetic recording disk and a slider containing a magnetic recording head to read and write the data. During operation, the disk is rotated by a centrally located motor running at between 7,200 and 15,000 rpm, while the slider flies over the disk bear by air within a few nanometers in order to detect the magnetic domain orientation. For a reliable operation of the slider-disk interface, SAMs of a perfluoropolyether (PTFE) lubricant are usually 
coated on the disk with a thickness of about $1 \mathrm{~nm}$ [113]. With the continual decrease in the head-disk clearance to increase the areal density, there is an increasing probability of lubricant transfer to the slider even in the absence of any head-disk contact, which is a severe hazard to the stable operation of the system and must therefore be avoided. The transfer mechanism is believed to involve lubricant evaporation from the disk surface and condensation onto the slider, the process of which can be reduced by decreasing the thickness of the coated SAMs, increasing the number of polar hydroxyl end groups per lubricant molecule, increasing the film bonding ratio or using lubricants with a stiffer backbone [114].

\subsection{Summary of boundary lubrication at the nanoscale}

Facilitated by the AFM and SFA with high precision, a number of advances in both theory and application have been achieved over the past several decades. With the arrival of nanotechnology, a new perspective of boundary lubrication at the nanoscale has come into view, and our understanding of boundary lubrication at the molecular level has been rapidly expanding. The ultra-low friction coefficient and stick-slip without wear are rarely observed in macroscopic experiments of boundary lubrication. This trend will continue owing to the relatively young age of the studies conducted in this area and the rich phenomena when scaling down to the molecular level. Because many complications in macroscopic systems, such as contact with multiple asperities, can be avoided, the relative simplicity of such a system also contributes to the elegant expression of these theories.

However, it may take a great deal of effort before the newly discovered knowledge regarding boundary lubrication at the nanoscale can be applied in real engineering of macroscopic systems. Connecting boundary lubrication at the nanoscale to the macroscopic scale presents a genuinely exciting and so far largely unexplored area of research. Based on the enormous number of studies conducted on the behaviors of boundary film at the nanoscale, it is time for scientists to reconsider the behaviors of boundary film in macroscopic systems, and the related boundary lubrication properties. New methodologies, theories, and experimental designs are needed in such studies to bridge the gap of boundary lubrication between the nanoscale and macroscopic scale.

\section{Active control of boundary lubrication}

\subsection{Significance of active control}

From the viewpoint of engineering, finding a way to control boundary lubrication is as important as an understanding of the mechanisms of boundary lubrication. The goal of active control of boundary lubrication is not only at controlling the magnitude of friction and wear [23], but also at controlling the distributions of friction and wear over the contact surfaces, as well as their variations over time [115]. Although lower friction and wear are beneficial in most engineering applications, proper levels of friction and wear are more preferable in many practical cases. For example, dangerous skidding will occur during high-speed rolling contact if the frictional traction force provided at the contact point is insufficiently high, such as in a roller-race contact of a rolling bearing, tire-road contact of a passenger car, or wheel-rail contact of a high-speed train. Bolt connections in a machine may become gradually loosened during operation if the static friction coefficient at the screw thread interface is too low. Zero-wear is also not the perfect case. A deliberate running-in process with controlled wear at the level of asperities of the mating surfaces is beneficial for all of the following machine operations. In some advanced surface finishing processes, such as lapping and chemical mechanical planarization (CMP), a proper wear rate is definitely required for maintaining an acceptable level of productivity $[116,117]$. In a machinery system, there are a number of contacting interfaces distributed throughout many locations, and different interfaces at the different locations of the machine have different roles and require different desired friction coefficient values. Therefore, it is meaningful to be able to finely tune the friction coefficient, not just the contact force, according to the design requirement for the specific function of each interface. For certain motion mechanisms, such as in a clutch, gecko-mimicked motion mechanism [118], or robot arm joint, a quick switch between a smooth 
motion and a reliable dead-locking is required. In the detachment and movement stage, lower kinetic friction and wear are preferable, while higher adhesion and static friction are better during the attachment and sticking stage, which means that friction should be changeable in a timely manner. Conventional boundary lubrication technologies are unable to fulfill such complicated tasks of controlling friction in magnitude, contacting position and time. Applying certain types of external fields provides the possibility to achieve the active control of boundary lubrication, the progress of which is described below.

\subsection{Potential controlled boundary lubrication in aqueous solutions}

Since the early use of electricity, people have dreamed of modulating friction by the means of electricity. In 1875, Edison patented a telegraphy device that utilized electricity to change the friction between metal and paper moistened with bromo chloralum or alcohol [119]. Although he claimed that the friction can be changed by the passage of electricity through the surfaces in contact, in his patent, he neither showed his experimental results nor gave an explanation of the possible mechanism of his findings. The first fundamental study on active control of boundary lubrication was conducted by Bowden and Young [120] in the early of 1950s. They introduced the latest potentiostatic technique developed in electrochemistry to the field of tribology, and found that the static coefficient of friction between a platinum rider and a platinum wire in a dilute solution of sulfuric acid depends on the relative interfacial potential of the platinum electrodes to a standard reference electrode. When the interfacial potential is within the region of either hydrogen or oxygen evolution, the static coefficient of friction is lower than that of an intermediate potential. The authors attributed the reduction in friction to the adsorption of hydrogen or oxygen gas film, which plays the role of boundary lubrication. Based on the progress made in the electrical doublelayer theory during the 1960s, some scientists tried to explain Bowden's experimental findings in terms of the difference in the repulsive force between electrical double layers on rubbing surfaces upon applying an electric potential [121], rather than the change in boundary film as suggested by Bowden. Such speculation of the electrical double-layer repulsive effect on friction was also adopted by Zhu et al. [122] in the early 1990s, and was used to analyze their experimental results obtained with iron/iron and iron oxide/alumina oxide rubbing contacts in $\mathrm{Na}_{2} \mathrm{SO}_{4}$ solution. However, their experimental results showed that the effect of interfacial potential on friction became weaker and weaker with an increase in the normal load when an inorganic electrolyte was used as lubricant. On the other hand, when a small amount of carboxylic acid was added into the aqueous solution, adjusting the interfacial potential within the range of -1.3 to $1.2 \mathrm{~V}$ versus a standard calomel electrode (SCE) resulted in a remarkable change in the coefficient of friction, from about 0.15 at a positive potential, to 0.31 at a potential more negative than $-0.8 \mathrm{~V}$, even under a relatively high contact pressure of $150 \mathrm{MPa}$. The authors reported that positive interfacial potentials produce iron carboxylate on the surfaces, thereby reducing friction significantly, whereas negative interfacial potentials give rise to a higher friction. During the same time period, Brandon et al. [123] also presented similar experimental curves of static coefficient of friction versus interfacial potential for iron upon contact with a mild steel in an aqueous solution containing octanoic acid under a light load with a tilting electrochemical cell. The authors presumed that the effect of interfacial potential on friction is the result of electrostatic interactions between the negatively charged octanoate species and the charged contact surfaces. In 2002, Chang et al. [124] found that the electrolysis of water might be the key trigger to the abrupt change in friction coefficient in the presence of a sufficiently negative electric potential because the transition from low to high friction in their test conditions was coincident with that of the electrolysis of the solution when adjusting electrode potential. They attributed the observed change in friction to the cleaning action of the local high $\mathrm{pH}$ value due to electrolysis under severely negative interfacial potentials for the laurylsulfonate species, which may form a good boundary lubricating film under positive or mildly negative potential conditions. These observations are consistent with the previous experimental results with similar aqueous lubrication [120-123]. 
To clarify which of the above-mentioned mechanisms, i.e., gas film lubrication owing to the electrolysis of water proposed by Bowden and Young [120], electrical double- layer repulsion proposed by Bockris and Argade [121], electrostatic interaction between the charged lubricious species and contact surfaces proposed by Brandon et al. [123], or cleaning of the surfactant film owing to the increased $\mathrm{pH}$ value through the electrolysis of the aqueous solution proposed by Chang et al. [124], is responsible for the observed potential-dependent change of friction coefficient in aqueous lubrication, experiments were repeatedly conducted with a refined adjustment of the concentration of sodium dodecyl sulfate (SDS) surfactant and the applied electrical potential. Meanwhile, electrochemical QCM technique was used to monitor the adsorption and desorption of the surfactant when the electrical potential was changed [23]. It was revealed that the electrolysis of a solution is not a necessary condition for the transition of friction to occur if the concentration of the surfactant additives is much lower than its critical micelle concentration (CMC) [23]. As shown in Fig. 16, for a blank solution, the measured friction coefficient under the same load and speed conditions is 0.45 , and is independent of the electrical potential. For the low concentration group (SDS concentration $<5 \mathrm{mM}$ ), friction transition occurs within the potential range of -0.4 to $0.05 \mathrm{~V}$, which is slightly different for different concentrations. It should be noted that, when the electrode potential is in the positive region, a layer of $\mathrm{DS}^{-}$anions is absorbed on stainless steel surface, as shown in Fig. 17, and the macroscopic friction is relatively low. On the other hand, when the potential is lower than $-0.4 \mathrm{~V}$, the $\mathrm{DS}^{-}$ anions desorb from the surface of the stainless steel, as shown in Fig. 17, and the macroscopic friction is relatively high. For the high concentration group (SDS concentration $>5 \mathrm{mM}$ ), however, the potential for friction transition shifts to the range of -1.2 to $-1.0 \mathrm{~V}$, which is close to the equilibrium potential for hydrogen evolution [124], implying that the desorption of DS $^{-}$anions and the hydrolysis of water proceed simultaneously under high SDS concentrations. Therefore, it can be concluded that the coefficient of friction can be controlled within the range from the low value in the presence of an adsorbed SDS layer

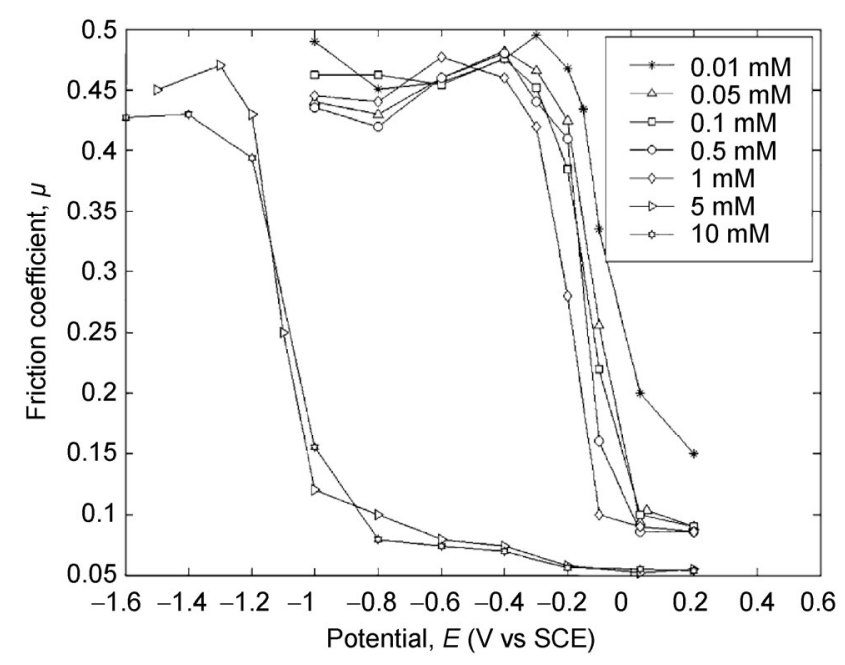

Fig. 16 Friction coefficient versus electrode potential in SDS solutions of different concentrations [23].

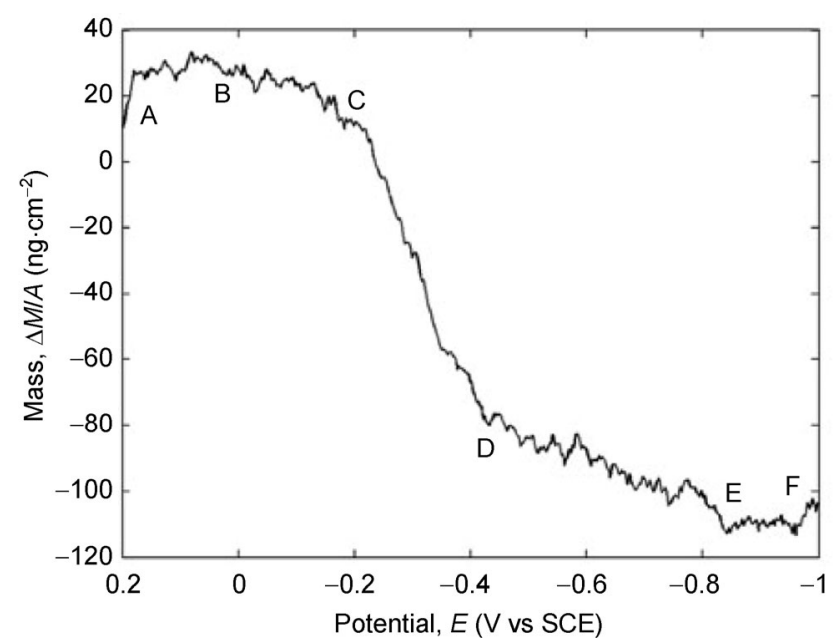

Fig. 17 Relationship between the adsorbed mass changes of SDS on stainless steel surface and the control potential for SDS solution with a concentration of $1 \mathrm{mM}$ using negative-going potential scanning from $0.2 \mathrm{~V}$ to $-0.1 \mathrm{~V}$ with a scan rate of $5 \mathrm{mV} / \mathrm{s}$ [23].

to the high value in the absence of the adsorbed SDS layer, depending on the polarity and magnitude of the charge on the contact surface. This provides a reasonable explanation for the potential-dependent friction phenomena from the standpoint of boundary lubrication.

Moreover, it was demonstrated that the friction coefficient can follow the change of the applied potential in any prescribed forms, such as a square wave, sinusoidal wave, or triangle wave, or in a manner of feedback control $[125,126]$. The response time of the friction to the potential is determined based on the charge/discharge time and mass transfer 
of the surfactant of the tribosystem. The control of local friction is also realizable by separating the contact surface into several zones with insulating layers and setting up different potential changes in different zones [115], or by using the bipolar technique with a uniform electrode.

\subsection{Potential controlled boundary lubrication in non-aqueous solutions}

The tunability of boundary lubrication of aqueous surfactant solutions described above is attractive because of the feasibility of smarter friction control. However, the application of an aqueous lubrication in industry is limited because of the drawbacks of a limited temperature range, evaporation, and corrosion against most metallic materials used in engineering, although it is dominant in biological systems. In recent years, an analogous potential effect on boundary lubrication in a non-aqueous system was found.

The primary difficulty in extending the potential controlled friction technique to a non-aqueous lubrication is in the high electrical resistivity of most lubricating oils, which makes the electrochemical techniques of monitoring and maintaining the electrode potentials suitable for aqueous solutions inapplicable to the situations in which oil lubrication is used. To overcome this problem, Zhu et al. [127] selected some polar fluids, including propylene carbonate (PC), tetrahydrofuran (THF), diethyl adipate (DEA), and butylbenzene (BB) as model base lubricants, and mixed them with three types of additives, ferrocene, dibenzyldisulfide, and carboxylic acids, with different chain lengths, respectively. The authors also added some supporting electrolytes to increase the conductivity of the non-aqueous fluids. Their experimental results indicate that the friction coefficient of iron-on-iron contact maintains a lower value in the negative potential range, and increases by $50 \%$ as the electrode potential is shifted from the rest potential of about $-0.1 \mathrm{~V}$ (SCE) to the positive potential of $1.5 \mathrm{~V}$ when lubricated using a $0.05 \mathrm{wt} \%$ octadecanoic acid solution in PC based fluid. The tendency of the change in friction with the electrode potential in the non-aqueous lubrication condition, however, is contrary to that they reported in aqueous lubrication condition [122]. Because no experimental data on the adsorption of boundary film under different electrode potentials are provided in $[122,123,127,128]$, the inconsistency of the potential-dependent lubrication behavior between the aqueous and non-aqueous carboxylic acid solutions cannot be satisfactorily explained solely from the effect of the electrode potential on the adsorption of carboxylic acid on iron surfaces.

Yang et al. [129] choose the SDS surfactant in a pure propylene carbonate based fluid as a model lubricant to investigate the effect of potential on friction. Propylene carbonate is a polar aprotic solvent and is frequently used as a high permittivity component of electrolytes in lithium batteries. Because of its high dielectric constant $\left(\varepsilon=65.0\right.$ at $\left.25^{\circ} \mathrm{C}\right)$, many types of nonorganic and organic salts, including the SDS surfactant, can be dissolved and dissociated in PC. No supporting electrolytes, except the SDS surfactant, were added to the solution to prevent the SDS boundary film from possible effects of ions. Water was also excluded from the solution, suppressing the effect of water on the SDS aggregate formation and the hydration effect on boundary lubrication. The boundary lubrication property of the SDS/PC solution within the potential range of $-1.5 \mathrm{~V}$ to $+1.5 \mathrm{~V}$ versus $\mathrm{Ag} / \mathrm{AgCl}$ was explored using a ball-on-disk friction tester. Meanwhile, the adsorption mass of SDS surfactant on stainless steel surface within the same potential range was measured using a quartz crystal microbalance (EC-QCM-D). The experimental results showed the potential-dependent changes in the boundary lubrication performance of the propylene carbonate solution. When the applied potential was positive, both the friction and wear of the tested stainless steels were relatively lower. As the potential was shifted to the negative regime, the friction coefficient increased by $100 \%$ or more, depending on the load condition and hardness of the stainless steels. The experimental results verify the coincident changes in the friction coefficient and the desorption amount of the SDS film, the mechanism of which is consistent with that found in the aqueous SDS solution. When the SDS surfactant was replaced with ionic liquids (ILs), 1-octyl3-methylimidazolium tetrafluoroborate ([OMIm] $\left.\mathrm{BF}_{4}\right)$, 1-octyl-3-methylimidazolium hexafluorophosphate ([OMIm] $\left.\mathrm{PF}_{6}\right)$, and 1-decyl-3-methylimidazolium hexafluorophosphate ([DMIm] $\left.\mathrm{PF}_{6}\right)$, a similar effect of the 
potential on boundary lubrication was found [129], suggesting that the adsorption of ions contributes greatly to boundary lubrication of the IL additives, and that the boundary lubricating ability can be improved to the utmost by applying a proper electrical potential to the steel surface. This mechanism even applies to pure IL as lubricant, with different types of adsorbed ion on the friction pair at different surface potentials [130].

Propylene carbonate is not the only candidate as the base fluid suitable for potential-dependent boundary lubrication. Other ester oils with polarity, such as diethyl succinate (DES), can also be effective when mixed with surfactants or ionic liquids. The results are similar to those found in PC solutions. This similarity confirms the mechanism of potential-dependent boundary lubrication for ester-based lubricants. In addition, it is not surprising to expect the similar potentialdependent boundary lubrication in a polyelectrolyte (PE)-lubricated tribosystem.

\subsection{Other approaches for active control of boundary lubrication}

One example of electric-field induced friction reduction and control in PE lubrication was reported by Drummond [131], who studied the interactions between molecularly smooth mica surfaces coated by PE brushes produced by self-assembly of amphiphilic diblock polystyrene-polyacrylic acid co-polymers (PS36-b-PAA125) from an aqueous solution at $\mathrm{pH} 10$, by using AFM and QCM in a liquid environment to confirm the adsorption of a $\mathrm{pH}$-responsive, nonstructured polymer layer, and measured the normal and lateral forces under electric field conditions using SFA. The adsorption of poorly solvated hydrophobic PS blocks (anchor) is driven by dispersion forces. On the contrary, the water soluble PAA moieties (buoy) are negatively charged at a $\mathrm{pH}$ above neutral, and are electrostatically repelled from the mica surfaces. Differing from the direct current electrical potential application mentioned in Sections 6.2 and 6.3, the author applied an alternating voltage between the lubricated surfaces instead. A remarkable friction reduction of up to $80 \%$ was observed with an increase in the amplitude of the alternating voltage. Furthermore, the friction reduction depends on not only the amplitude but also the frequency of the alternating voltage. A substantial reduction in friction can be observed when the frequency is lower than $1 \mathrm{kHz}$, but no effect is detectable at larger frequencies. In addition, the friction reduction effect is considerably lessened within a narrow frequency window of around $500 \mathrm{~Hz}$. Below this frequency, the effect is quite substantial, whereas at larger frequencies it is progressively diminished. The author described the frequency dependence of friction in terms of the dynamic conformal change of PE ionization under the electric field. Recently, Strelcov et al. reported that boundary lubrication properties can be actively controlled between an AFM tip and a salt surface when the relative humidity is above a certain threshold and a sufficiently strong electric field is applied [132]. A plausible mechanism was proposed that an ordered structure of electric double layer as a result of water condensation and dissolution of polarizable ions accounts for the reduction of friction force.

Another way to control the boundary lubrication is to modulate the inherent surface forces, including shortrange van der Waals forces and long-range Casimir force, within the range of attractive to repulsive values by means of an external field. Since the Casimir force is dependent on the permeability of the interacting objects that can be controlled by an external magnetic field, it is applicable to adjust the Casimir force by this field. However, for most magnetic materials with low frequency magnetic responses, the adjustment range is insignificant. Recently, Ma et al. proposed a superparamagnetic metameta-material (MMM) to realize nontrivial, high-frequency permeability as well as small permittivity to obtain a repulsive Casimir force between the MMM plate and a metal plate [133]. The authors predicted that the Casimir force can be successfully adjusted between repulsion and attraction continuously by an external magnetic field.

Magnetic fluid (or magnetorheological fluid) is another system that can be actively controlled by external magnetic field, which is a colloid suspension of single-domain ferromagnetic particles dispersed in a carrier liquid. Using magnetic fluid as lubricant, the friction coefficient in boundary lubrication regime can be magnified four-fold when a certain external magnetic field is applied in the four-ball tribological 
tester reported by $\mathrm{Hu}$ et al. [134]. Using a magnetic surface, Chen et al. designed a lubricating system in which magnetic fluid exhibits better lubricating properties with an applied magnetic field than without such a field, by forming a magnetically arrayed film on the surface [135]. As a type of electromagnetic wave, UV light was also used to achieve active control of boundary lubrication. In such a system, chiral-nematic polymer-network coating containing photosensitive azobenzene molecules acts as boundary film, the conformation of which is sensitive to radiated UV light, accounting for the light controlled boundary lubrication properties. There is also evidence that boundary lubrication properties can be regulated by changing the solvent, electrolyte, $\mathrm{pH}$, and temperature of the solution, reviewed in Ref. [136]; however, these methods cannot be deemed an active control of boundary lubrication when compared to previous discussed methods because a fast yet reversible control of boundary lubrication properties is not easy to achieve.

\subsection{Summary of the active control of boundary lubrication}

Active control represents the future of boundary lubrication, although studies on it are still at a premature stage, and the technology is far from reaching the industrial application stage. The achievements in this area mainly include the revealing of the active control mechanisms in different systems, which was comprehensively reviewed in this section. Control of boundary lubrication by potential remains the most convenient and intensively investigated type of active control [136], the mechanism of which is summarized in Fig. 18. Generally speaking, using anionic surfactant as lubricating additive, the friction coefficient is low lubricated by the adsorption film at a relatively positive applied potential, and is high for a relatively negative applied potential as the adsorption film is desorbed by electrostatic repulsion. The critical potential for the transition of friction coefficient varies with the concentration of the surfactant, as the adsorption amount and adsorption structure change, as well as the response of adsorption film to applied potential, as illustrated in Fig. 18. This mechanism provides a model lubricating system, using water or oil as base lubricant, with the additive of surfactants or ionic liquids, the boundary lubrication effect of which can be remarkably controlled at applied surface potential. New methods for the active control of boundary lubrication are also emerging, such as a boundary lubricating system of polyelectrolyte, pure IL, a salt surface in a vapor environment, photosensitive polymer-network, or magnetic fluid, each of which has its own unique properties in response to an external field. Compared to the enormous effort in

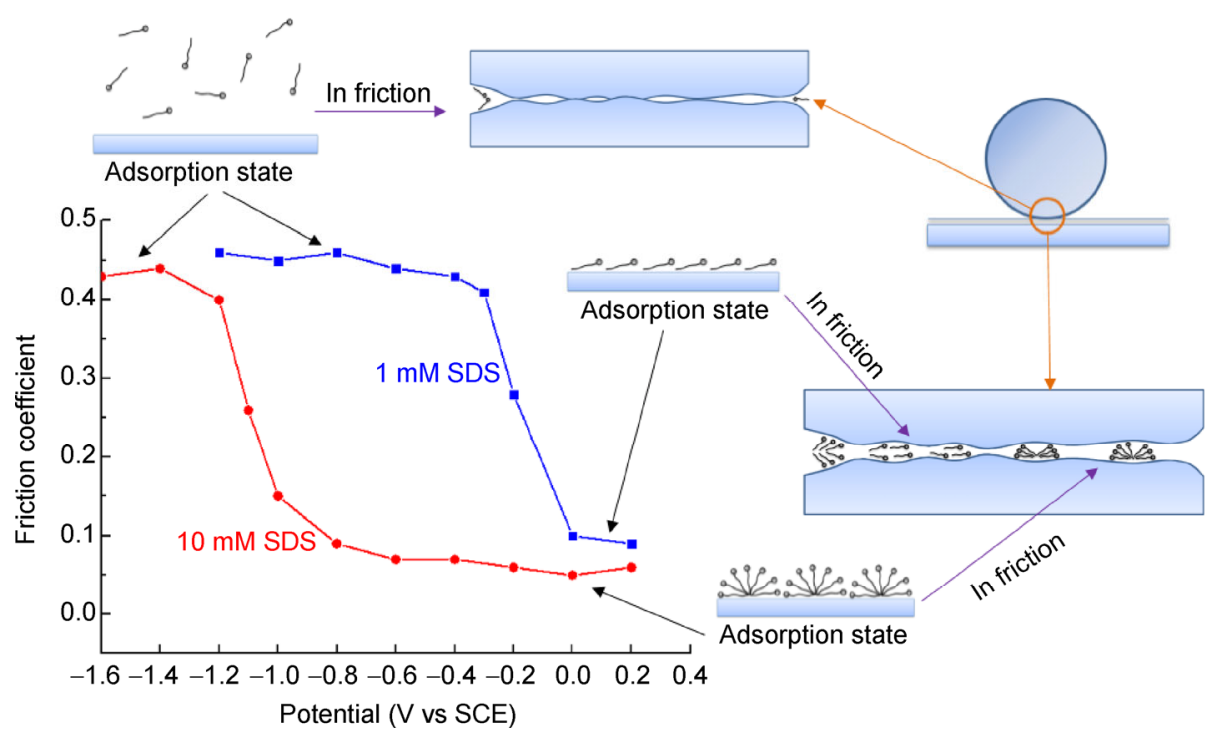

Fig. 18 Mechanism of potential controlled friction with adsorbed boundary film. 
illustrating the nature of boundary lubrication, the active control of boundary lubrication is a relatively new area that should be given more attention. The developing trends in such a new field are further prospected in the conclusion of this review.

\section{Conclusions and remarks}

The present review on boundary lubrication by adsorption film is unable to include all knowledge regarding this vigorous field that has been uncovered by scientists during the past century, but does provide a view of the mainline of leading perspectives and methodologies in revealing the fundamental problems of boundary lubrication. Unlike some areas in natural science such as electromagnetism, in which the abundant phenomena involved can be fully revealed by the elegant Maxwell's equations, no simple conclusions can be drawn up in the research on boundary lubrication. Whenever one boundary lubrication model has been established based on an enormous number of experiments using a particular system, strong evidence would emerge indicating that the model was far from satisfactory in fully revealing the nature of boundary lubrication. The complexity of boundary lubrication system is the main reason for the inherent difficulties in related studies, but is also its amazement in attracting the attention of a significant number of researchers ever since it was categorized as an independent lubrication regime. Generally speaking, most research on boundary lubrication has been conducted experimentally, as a quantitative prediction of the friction and wear at boundary lubrication is difficult to achieve. Thus, our understanding of boundary lubrication, or the ability to model boundary lubrication in theory, depends greatly on the experimental facilities available in the contemporary laboratory. For example, based on his meticulous investigation using his developed macroscopic friction tester, Bowden's model dominated the interpretation of boundary lubrication for decades, and a real look into the boundary lubrication properties at the nanoscale was only made after sufficiently precise equipment such as SFA and AFM were invented. Thanks to the amplification in the number of modern techniques available for not only boundary lubrication studies but also for characterizing the properties of boundary film, an elaboration on the correlation between the boundary lubrication performance and the behaviors of boundary film during the sliding process has been an efficient protocol in revealing more detailed aspects of the boundary lubrication process.

One central topic has dominated the past discussion of the nature of boundary lubrication, and will likely remain an area of focus in future research, i.e., how to link the macroscopic boundary lubrication properties with its functioning process at the nanoscale. As early as the first study on boundary lubrication, conducted by Hardy, the main strategy was trying to illustrate the boundary lubrication properties at the macro scale by a speculated model mainly involving the interaction of boundary films at the molecular level. Later, Bowden's model emphasized the role of direct contact and adhesion between lubricated sliding surfaces, and used a parameter $\alpha$ to generalize the breakdown ratio of boundary film. This framework in understanding the process of boundary lubrication is simple yet efficient from a macroscopic perspective, but ignores the real distribution of the areas where breakdown of the boundary film occurs and the behaviors of boundary film during the sliding process. Fortunately, the behaviors of boundary film during the sliding process have been intensively investigated by AFM and SFA over the past several decades, through which the behaviors of boundary film can be surveyed directly at the molecular level. However, in the boundary lubrication process at the macroscopic level, the behaviors of the boundary film may be related to but still differ from those under an ideal single asperity contact at the nanoscale. Although we are very close, we still have not uncovered the complex multi-scale process that occurs during boundary lubrication at the macroscopic level. The lubrication process is macroscopic as a whole, whereas the basic volume of a material affected through contact tends to be extremely small, even at the nanoscale, with an uneven distribution of factors such as the contact pressure and temperature, and the changing of these factors during the sliding process. Statistical mechanics, which is efficient at connecting macroscopic quantity with micro molecular motion in systems such as a gas, may also play a part in solving a similar problem 
for boundary lubrication, at least in relating the breakdown ratio of boundary film with the distribution function of the contact pressure and temperature, as one simple example. There have also been attempts at correlating the boundary lubrication properties at the macroscopic level for sliding rough surfaces with the behavior of lubricant molecules by combining phase transition model with thermodynamic method [137]. A multi-scale analysis of the interfacial interactions has also been employed to determine the initiation of instable wear of boundary lubrication [138].

As engineering science, despite the abundant theories illustrating the complicated process of boundary lubrication, the ultimate goal of this research topic is to efficiently control the boundary lubrication properties so as to accommodate its application in machinery systems. During the past century, the main focus of attention was on the synthesis and characterization of lubricating additives, which easily achieved an application in engineering systems and in the marketplace. However, there is no denying that the mechanisms of active control of boundary lubrication in certain model lubricating systems have been nearly clarified. For example, water or oil based lubricating system, with the additive of surfactants or ionic liquids, can be actively controlled under the potential of a direct or alternating voltage as a response of boundary film to applied field. It is expected that lubricating systems that are able to be actively controlled can be widely expanded, at least, but not limited to, such constitution of the existing model systems. New mechanisms and model systems that may have a better performance will also be discovered. The general goal of an active control system is to achieve regulation of tribology properties at a larger magnitude and more quickly. To find its application in industry, for example, in an automobile clutch, other factors such as the stability, compatibility, and economic efficiency will also have to be taken into consideration. Moreover, the goal of active control of boundary lubrication is not only controlling the magnitude of the friction and wear, but also controlling their distribution over a contact surface, which may also find an industrial application. As prospects in the research on boundary lubrication, the trends listed herein are not comprehensive, but represent several important and meaningful breakthroughs that may be achieved in the near future.

\section{Acknowledgements}

This work was supported by the National Natural Science Foundation of China (NSFC) with the grant No. 91123033.

Open Access: This article is distributed under the terms of the Creative Commons Attribution License which permits any use, distribution, and reproduction in any medium, provided the original author(s) and source are credited.

\section{References}

[1] Dorinson A, Ludema K C. Mechanics and Chemistry in Lubrication. New York: Elsevier Science Publishing Company, 1985.

[2] Szeri A Z. Fluid Film Lubrication: Theory and Design. Cambridge: Cambridge University Press, 1998.

[3] Pitenis A A, Dowson D, Gregory Sawyer W. Leonardo da Vinci's friction experiments: An old story acknowledged and repeated. Tribol Lett 56(3): 509-515 (2014)

[4] Barnes A M, Bartle K D, Thibon V R A. A review of zinc dialkyldithiophosphates (ZDDPS): Characterisation and role in the lubricating oil. Tribol Int 34(6): 389-395 (2001)

[5] Spikes H. The history and mechanisms of ZDDP. Tribol Lett 17(3): 469-489 (2004)

[6] Nicholls M A, Do T, Norton P R, Kasrai M, Bancroft G M. Review of the lubrication of metallic surfaces by zinc dialkyl-dithiophosphates. Tribol Int 38(1): 15-39 (2005)

[7] Hardy W B. Boundary lubrication - The paraffin series. Proc $R$ Soc Lond A 100(707): 550-574 (1922)

[8] Gibbs R E. An X-ray investigation of the lower members of the fatty acid series. J Chem Soc 125(2): 2622-2625 (1924)

[9] Cameron A. A theory of boundary lubrication. ASLE Trans 2(2): 195-198 (1959)

[10] Langmuir I. Mechanical properties of monomolecular films. Journal of the Frankl Inst 218: 143-171 (1934)

[11] Bowden F P, Leben L. The friction of lubricated metals. Philos T R Soc A 239(799): 1-27 (1940)

[12] Park S, Kim Y W, Lim J C, Ahn H S, Park S J. Nano- and microscale friction behaviors of functionalized self-assembled monolayers. J Ind Eng Chem 9(1): 16-24 (2003) 
[13] Gosvami N N, Bares J A, Mangolini F, Konicek A R, Yablon D G, Carpick R W. Mechanisms of antiwear tribofilm growth revealed in situ by single-asperity sliding contacts. Science 348(6230): 102-106 (2015)

[14] Lee S, Shon Y, Colorado R, Guenard R L, Lee T R, Perry S S. The influence of packing densities and surface order on the frictional properties of alkanethiol self-assembled monolayers (SAMs) on gold: A comparison of SAMs derived from normal and spiroalkanedithiols. Langmuir 16(5): 2220-2224 (2000)

[15] Clear S C, Nealey P F. Lateral force microscopy study of the frictional behavior of self-assembled monolayers of octadecyltrichlorosilane on silicon/silicon dioxide immersed in n-alcohols. Langmuir 17(3): 720-732 (2001)

[16] Atkin R, Craig V, Wanless E J, Biggs S. Mechanism of cationic surfactant adsorption at the solid-aqueous interface. Advances in Colloid and Interface Science 103(3): 219-304 (2003)

[17] Paria S, Khilar K C. A review on experimental studies of surfactant adsorption at the hydrophilic solid-water interface. Adv Colloid Interface Sci 110(3): 75-95 (2004)

[18] Chen M, Burgess I, Lipkowski J. Potential controlled surface aggregation of surfactants at electrode surfaces-A molecular view. Surf Sci 603(10-12): 1878-1891 (2009)

[19] Clark S C, Ducker W A. Exchange rates of surfactant at the solid-liquid interface obtained by ATR-FTIR. J Phys Chem B 107(34): 9011-9021 (2003)

[20] Schniepp H C, Saville D A, Aksay I A. Self-healing of surfactant surface micelles on millisecond time scales. $J \mathrm{Am}$ Chem Soc 128(38): 12378-12379 (2006)

[21] Boschkova K, Kronberg B, Stålgren J J R, Persson K, Salagean M R. Lubrication in aqueous solutions using cationic surfactants - A study of static and dynamic forces. Langmuir 18(5): 1680-1687 (2002)

[22] Sulek M W, Wasilewski T, Kurzydłowski K J. The effect of concentration on lubricating properties of aqueous solutions of sodium lauryl sulfate and ethoxylated sodium lauryl sulfate. Tribol Lett 40(3): 337-345 (2010)

[23] He S, Meng Y, Tian Y. Correlation between adsorption/ desorption of surfactant and change in friction of stainless steel in aqueous solutions under different electrode potentials. Tribol Lett 41(3): 485-494 (2011)

[24] Zhang J, Meng Y. Stick-slip friction of stainless steel in sodium dodecyl sulfate aqueous solution in the boundary lubrication regime. Tribol Lett 56(3): 543-552 (2014)

[25] Feiler A, Plunkett M A, Rutland M W. Atomic force microscopy measurements of adsorbed polyelectrolyte layers. 1. dynamics of forces and friction. Langmuir 19(10): 4173-4179 (2003)
[26] Plunkett M A, Feiler A, Rutland M W. Atomic force microscopy measurements of adsorbed polyelectrolyte layers. 2. effect of composition and substrate on structure, forces, and friction. Langmuir 19(10): 4180-4187 (2003)

[27] Yan X, Perry S S, Spencer N D, Pasche S, De Paul S M, Textor M, Lim M S. Reduction of friction at oxide interfaces upon polymer adsorption from aqueous solutions. Langmuir 20(2): 423-428 (2004)

[28] Raviv U, Tadmor R, Klein J. Shear and frictional interactions between adsorbed polymer layers in a good solvent. $J$ Phys Chem B 105(34): 8125-8134 (2001)

[29] Hsiao E, Bradley L C, Kim S H. Improved substrate protection and self-healing of boundary lubrication film consisting of polydimethylsiloxane with cationic side groups. Tribol Lett 41(1): 33-40 (2011)

[30] Wangchareansak T, Craig V S J, Notley S M. Adsorption isotherms and structure of cationic surfactants adsorbed on mineral oxide surfaces prepared by atomic layer deposition. Langmuir 29(48): 14748-14755 (2013)

[31] Manne S, Gaub H E. Molecular organization of surfactants at solid-liquid interfaces. Science 270(5241): 1480-1482 (1995)

[32] Burgess I, Jeffrey C A, Cai X, Szymanski G, Galus Z, Lipkowski J. Direct visualization of the potential-controlled transformation of hemimicellar aggregates of dodecyl sulfate into a condensed monolayer at the $\mathrm{Au}(111)$ electrode surface. Langmuir 15(8): 2607-2616 (1999)

[33] Schniepp H C, Shum H C, Saville D A, Aksay I A. Surfactant aggregates at rough solid-liquid interfaces. $J$ Phys Chem B 111(30): 8708-8712 (2007)

[34] Brosseau C L, Sheepwash E, Burgess I J, Cholewa E, Roscoe S G, Lipkowski J. Adsorption of N-Decyl-N,N,Ntrimethylammonium triflate (DeTATf), a cationic surfactant, on the $\mathrm{Au}(111)$ electrode surface. Langmuir 23(4): 1784-1791 (2007)

[35] Soares D M, Gomes W E, Tenan M A. Sodium dodecyl sulfate adsorbed monolayers on gold electrodes. Langmuir 23(8): 4383-4388 (2007)

[36] Karlsson P M, Palmqvist A E C, Holmberg K. Adsorption of sodium dodecyl sulfate and sodium dodecyl phosphate on aluminum, studied by QCM-D, XPS, and AAS. Langmuir 24(23): 13414-13419 (2008)

[37] Lu G, Gillece T W, Moore D J. Study of water vapor and surfactant absorption by lipid model systems using the quartz crystal microbalance. Chem Phys Lipids 164(4): 259-265 (2011)

[38] Duan M, Wang H, Fang S, Liang Y. Real-time monitoring the adsorption of sodium dodecyl sulfate on a hydrophobic surface using dual polarization interferometry. $J$ Colloid Interf Sci 417: 285-292 (2014) 
[39] Burgess I, Zamlynny V, Szymanski G, Lipkowski J, Majewski J, Smith G, Satija S, Ivkov R. Electrochemical and neutron reflectivity characterization of dodecyl sulfate adsorption and aggregation at the gold-water interface. Langmuir 17(11): 3355-3367 (2001)

[40] Simič R, Kalin M, Hirayama T, Korelis P, Geue T. Fatty acid adsorption on several DLC coatings studied by neutron reflectometry. Tribol Lett 53(1): 199-206 (2014)

[41] Kalin M, Simič R, Hirayama T, Geue T, Korelis P. Neutronreflectometry study of alcohol adsorption on various DLC coatings. Appl Surf Sci 288: 405-410 (2014)

[42] Zaera F. Probing liquid/solid interfaces at the molecular level. Chem Rev 112(5): 2920-2986 (2012)

[43] Bowden F P. Friction. Nature 166: 330-334 (1950)

[44] Hsu S, Ying C, Zhao F. The nature of friction: A critical assessment. Friction 2(1): 1-26 (2014)

[45] Greenwood J A, Williamson J B P. Contact of nominally flat surfaces. Proc R Soc Lond A 295(1442): 300-319 (1966)

[46] Whitehou. D J, Archard J F. The properties of random surfaces of significance in their contact. Proc R Soc Lond A 316(1524): 97 (1970)

[47] Bowden F P, Tabor D. The lubrication by thin metallic films and the action of bearing metals. J Appl Phys 14(3): 141 (1943)

[48] Bowden F P, Moore A J W, Tabor D. The ploughing and adhesion of sliding metals. J Appl Phys 14(2): 80 (1943)

[49] Bowden F P, Gregory J N, Tabor D. Lubrication of metal surfaces by fatty acids. Nature 156(3952): 97-101 (1945)

[50] Lee D W, Banquy X, Israelachvili J N. Stick-slip friction and wear of articular joints. Proc Nat Acad Sci 110(7): E567-E574 (2013)

[51] Galvanetto U, Bishop S R, Briseghella L. Mechanical stickslip vibrations. Int J Bifurcat Chaos 5(3): 637-651 (1995)

[52] Karnopp D. Computer simulation of stick-slip friction in mechanical dynamic systems. J Dyn Syst Meas Control 107(1): 100-103 (1985)

[53] Johnson P A, Savage H, Knuth M, Gomberg J, Marone C. Effects of acoustic waves on stick-slip in granular media and implications for earthquakes. Nature 451(7174): 57-60 (2008)

[54] Walker D M, Tordesillas A, Small M, Behringer R P, Tse C K. A complex systems analysis of stick-slip dynamics of a laboratory fault. Chaos 24(1): 13132 (2014)

[55] Wojewoda J, Stefanski A, Wiercigroch M, Kapitaniak T. Hysteretic effects of dry friction: modelling and experimental studies. Philos T R Soc A 366(1866): 747-765 (2008)

[56] Saha A, Wahi P. An analytical study of time-delayed control of friction-induced vibrations in a system with a dynamic friction model. Int J Nonlin Mech 63: 60-70 (2014)
[57] Wang D W, Mo J L, Ouyang H, Chen G X, Zhu M H, Zhou $Z$ R. Experimental and numerical studies of friction-induced vibration and noise and the effects of groove-textured surfaces. Mech Syst Signal Process 46(2): 191-208 (2014)

[58] Yoshizawa H, Israelachvili J. Fundamental mechanisms of interfacial friction. 2. stick-slip friction of spherical and chain molecules. J Phys Chem 97(43): 11300-11313 (1993)

[59] Kramer I R, Denier L J. Effects of environment on mechanical properties of metals. Prog Mater Sci 9(3): 131-199 (1961)

[60] Buckley D H. Effect of surface films on deformation of zinc single-crystal surface during sliding. ASLE Trans 15(2): 96-102 (1972)

[61] Buckley D H. Surface Effects in Adhesion, Friction, Wear, and Lubrication. New York: Elsevier Scientific Publishing Company, 1981.

[62] Bosman R, Hol J, Schipper D J. Running-in of metallic surfaces in the boundary lubrication regime. Wear 271(7-8): 1134-1146 (2011)

[63] Bowden F P, Tabor D. The Friction and Lubrication of Solids. Oxford: Clarendon Press, 1950.

[64] Archard J F. Contact and rubbing of flat surfaces. J Appl Phys 24(8): 981 (1953)

[65] Mcfarlane J S, Tabor D. Adhesion of solids and the effect of surface films. Philos $T R$ Soc A 202(1069): 224-243 (1950)

[66] Beerbower A. Boundary lubrication-scientific and technical forecast report. US Army Report AD747336, 1972.

[67] Kingsbury E P. Some aspects of the thermal desorption of a boundary lubricant. J Appl Phys 29(6): 888 (1958)

[68] Rowe C N. Some aspects of the heat of adsorption in the function of a boundary lubricant. ASLE Trans 9(1): 101-111 (1966)

[69] Wang W, Huang P. The calculation model of boundary lubrication under point contact. In Proceedings of ASME/STLE 2007 International Joint Tribology Conference, Parts A and B, 2007: 85-87.

[70] Adamson A W. Physical Chemistry of Surfaces. 3ed. New York: Interscience, 1976.

[71] Tabor D. The role of surface and intermolecular forces in thin film lubrication. In Microscopic Aspects of Adhesion and Lubrication Proceedings of the 34th International Meeting of the Société de Chimie Physique. Georges J M, Ed. New York: Elsevier, 1981: 651-682.

[72] Homola A M, Israelachvili J N, Gee M L, McGuiggan P M. Measurements of and relation between the adhesion and friction of two surfaces separated by molecularly thin liquid films. J Tribol 111(4): 675 (1989) 
[73] Hu Y, Ma T, Wang H. Energy dissipation in atomic-scale friction. Friction 1(1): 24-40 (2013)

[74] Mate C M, McClelland G M, Erlandsson R, Chiang S. Atomic-scale friction of a tungsten tip on a graphite surface. Phys Rev Lett 59(17): 1942-1945 (1987)

[75] Gane N, Bowden F P. Microdeformation of solids. J Appl Phys 39(3): 432-435 (1968)

[76] Szlufarska I, Chandross M, Carpick R W. Recent advances in single-asperity nanotribology. J Phys D: Appl Phys 41(12): 123001 (2008)

[77] Bhushan B, Israelachvili J N, Landman U. Nanotribology: Friction, wear and lubrication at the atomic scale. Nature 374: 607-616 (1995)

[78] Binnig G, Quate C F, Gerber C. Atomic force microscope. Phys Rev Lett 56(9): 930-933 (1986)

[79] Binnig G, Rohrer H, Gerber C, Weibel E. Surface studies by scanning tunneling microscopy. Phys Rev Lett 49(1): 57-61 (1982)

[80] Meyer G, Amer N M. Simultaneous measurement of lateral and normal forces with an optical-beam-deflection atomic force microscope. Appl Phys Lett 57(20): 2089-2091 (1990)

[81] Butt H, Cappella B, Kappl M. Force measurements with the atomic force microscope: Technique, interpretation and applications. Surf Sci Rep 59(1-6): 1-152 (2005)

[82] Park J Y, A T P. Atomic scale friction and adhesion properties of quasicrystal surfaces. J Phys: Condensed Matter 20(31): 314012 (2008)

[83] Tabor D, Winterton R H S. The direct measurement of normal and retarded van der waals forces. Proc $R$ Soc Lond A 312(1511): 435-450 (1969)

[84] Huang J, Yan B, Faghihnejad A, Xu H, Zeng H. Understanding nanorheology and surface forces of confined thin films. Korea-Aust Rheol J 26(1): 3-14 (2014)

[85] Park J Y, Salmeron M. Fundamental aspects of energy dissipation in friction. Chem Rev 114(1): 677-711 (2014)

[86] Yoshizawa H, Chen Y L, Israelachvili J. Fundamental mechanisms of interfacial friction. 1. Relation between adhesion and friction. J Phys Chem 97(16): 4128-4140 (1993)

[87] Leitch J J, Collins J, Friedrich A K, Stimming U, Dutcher J R, Lipkowski J. Infrared studies of the potential controlled adsorption of sodium dodecyl sulfate at the $\mathrm{Au}(111)$ electrode surface. Langmuir 28(5): 2455-2464 (2012)

[88] Gee M L, Mcguiggan P M, Israelachvili J N, Homola A M. Liquid to solidlike transitions of molecularly thin films under shear. J Chem Phys 93(3): 1895-1906 (1990)

[89] Klein J, Kumacheva E. Confinement-induced phase transitions in simple liquids. Science 269(5225): 816-819 (1995)

[90] Thompson P A, Robbins M O. Origin of stick-slip motion in boundary lubrication. Science 250(4982): 792-794 (1990)
[91] Lyashenko I A. First-order phase transition between the liquidlike and solidlike structures of a boundary lubricant. Tech Phys 57(1): 17-26 (2012)

[92] Ruths M, Israelachvili J N. Surface forces and nanorheology of molecularly thin films. In Nanotribology and Nanomechanics. Bhushan B, Ed. Berlin Heidelberg: Springer-Verlag, 2011: 107-202.

[93] Jagla E A. Boundary lubrication properties of materials with expansive freezing. Phys Rev Lett 88: 24550424 (2002)

[94] Raviv U, Klein J. Fluidity of bound hydration layers. Science 297(5586): 1540-1543 (2002)

[95] Raviv U, Perkin S, Laurat P, Klein J. Fluidity of water confined down to subnanometer films. Langmuir 20(13): 5322-5332 (2004)

[96] Raviv U, Giasson S, Kampf N, Gohy J, Jérôme R, Klein J. Lubrication by charged polymers. Nature 425(6954): 163-165 (2003)

[97] Briscoe W H, Titmuss S, Tiberg F, Thomas R K, McGillivray D J, Klein J. Boundary lubrication under water. Nature 444(7116): 191-194 (2006)

[98] Trunfio-Sfarghiu A, Berthier Y, Meurisse M, Rieu J. Role of nanomechanical properties in the tribological performance of phospholipid biomimetic surfaces. Langmuir 24(16): 8765-8771 (2008)

[99] Seror J, Sorkin R, Klein J. Boundary lubrication by macromolecular layers and its relevance to synovial joints. Polym Adv Technol 25(5): 468-477 (2014)

[100] Klein J. Hydration lubrication. Friction 1(1): 1-23 (2013)

[101] Garrec D A, Norton I T. Boundary lubrication by sodium salts: A Hofmeister series effect. J Colloid Interf Sci 379(1): 33-40 (2012)

[102] Wei Q, Cai M, Zhou F, Liu W. Dramatically tuning friction using responsive polyelectrolyte brushes. Macromolecules 46(23): 9368-9379 (2013)

[103] Bhushan B, Liu H W. Nanotribological properties and mechanisms of alkylthiol and biphenyl thiol self-assembled monolayers studied by AFM. Phys Rev B 63(24541224) (2001)

[104] Xiao X, Hu J, Charych D H, Salmeron M. Chain length dependence of the frictional properties of alkylsilane molecules self-assembled on mica studied by atomic force microscopy. Langmuir 12(2): 235-237 (1996)

[105] McDermott M T, Green J, Porter M D. Scanning force microscopic exploration of the lubrication capabilities of n-alkanethiolate monolayers chemisorbed at gold structural basis of microscopic friction and wear. Langmuir 13(9): 2504-2510 (1997)

[106] Li L Y, Yu Q M, Jiang S Y. Quantitative measurements of frictional properties of n-alkanethiols on $\mathrm{Au}(111)$ by scanning 
force microscopy. J Phys Chem B 103(39): 8290-8295 (1999)

[107] Sambasivan S, Hsieh S, Fischer D A, Hsu S M. Effect of self-assembled monolayer film order on nanofriction. $J$ Vac Sci Technol A 24(4): 1484-1488 (2006)

[108] Zheng X, Zhu H, Kosasih B, Kiet Tieu A. A molecular dynamics simulation of boundary lubrication: The effect of n-alkanes chain length and normal load. Wear 301(1-2): 62-69 (2013)

[109] Zhang Q, Archer L A. Interfacial friction of surfaces grafted with one- and two-component self-assembled monolayers. Langmuir 21(12): 5405-5413 (2005)

[110] Shen S, Meng Y, Zhang W. Characteristics of the wear process of side-wall surfaces in bulk-fabricated Si-MEMS devices in nitrogen gas environment. Tribol Lett 47(3): 455-466 (2012)

[111] Shen S, Meng Y. Adhesive and corrosive wear at microscales in different vapor environments. Friction 1(1): 72-80 (2013)

[112] Patton S T, Cowan W D, Eapen K C, Zabinski J S. Effect of surface chemistry on the tribological performance of a MEMS electrostatic lateral output motor. Tribol Lett 9(3): 199-209 (2000)

[113] Li N, Zheng L, Bogy D B, Meng Y. Flyability and durability test of dynamic fly-height sliders at 1-nm clearance. Tribol Trans 53(2): 212-218 (2010)

[114] Li N, Meng Y, Bogy D B. Effects of PFPE lubricant properties on the critical clearance and rate of the lubricant transfer from disk surface to slider. Tribol Lett 43(3): 275-286 (2011)

[115] Meng Y, Hu B, Chang Q. Control of local friction of metal/ceramic contacts in aqueous solutions with an electrochemical method. http://linkinghub.elsevier.com/ retrieve/pii/S0043164805003807, 2006.

[116] Kwon T, Ramachandran M, Park J. Scratch formation and its mechanism in chemical mechanical planarization (CMP). Friction 1(4): 279-305 (2013)

[117] Zhao D, Lu X. Chemical mechanical polishing: Theory and experiment. Friction 1(4): 306-326 (2013)

[118] Zhou M, Pesika N, Zeng H, Tian Y, Israelachvili J. Recent advances in gecko adhesion and friction mechanisms and development of gecko-inspired dry adhesive surfaces. Friction 1(2): 114-129 (2013)

[119] Edison T. Improvement in telegraph apparatus. Patent U. S. 158787, 1875.

[120] Bowden F P, Young L. Influence of interfacial potential on friction and surface damage. Research 3(5): 235-237 (1950)

[121] Bockris J O, Argade S D. Dependence of friction at wet contacts upon interfacial potential. J Chem Phys 50(4): 1622-1623 (1969)
[122] Zhu Y Y, Kelsall G H, Spikes H A. The influence of electrochemical potentials on the friction and wear of the friction and wear of iron and iron oxides in aqueous systems. Tribol Trans 37(4): 811-819 (1994)

[123] Brandon N P, Bonanos N, Fogarty P O, Mahmood M N, Moore A J, Wood R J K. Influence of potential on the friction and wear of mild steel in a model aqueous lubricant. $J$ Appl Electrochem5(23): 456-462 (1993)

[124] Chang Q Y, Meng Y G, Wen S Z. Influence of interfacial potential on the tribological behavior of brass/silicon dioxide rubbing couple. Appl Surf Sci 202(1-2): 120-125 (2002)

[125] Meng Y, Jiang H, Wong P L. An experimental study on voltage-controlled friction of alumina/brass couples in zinc stearate/water suspension. Tribol Trans 44(4): 567-574 (2001)

[126] He S, Meng Y, Tian Y, Zuo Y. Response characteristics of the potential-controlled friction of $\mathrm{ZrO}_{2} /$ stainless steel tribopairs in sodium dodecyl sulfate aqueous solutions. Tribol Lett 38(2): 169-178 (2010)

[127] Zhu Y, Ogano S, Kelsall G, Spikes H A. The study of lubricant additive reactions using non-aqueous electrochemistry. Tribol Trans 43(2): 175-186 (2000)

[128] Brandon N P. The effect of interfacial potential on friction in a model aqueous lubricant. $J$ Electrochem Soc 139(12): 3489 (1992)

[129] Yang X, Meng Y, Tian Y. Effect of imidazolium ionic liquid additives on lubrication performance of propylene carbonate under different electrical potentials. Tribol Lett 56(1): 161-169 (2014)

[130] Sweeney J, Hausen F, Hayes R, Webber G B, Endres F, Rutland M W, Bennewitz R, Atkin R. Control of nanoscale friction on gold in an ionic liquid by a potential-dependent ionic lubricant layer. Physl Rev Lett 109(15): 155502 (2012)

[131] Drummond C. Electric-field-induced friction reduction and control. Phys Rev Lett 109(15): 154302 (2012)

[132] Strelcov E, Kumar R, Bocharova V, Sumpter B G, Tselev A, Kalinin S V. Nanoscale lubrication of ionic surfaces controlled via a strong electric field. Sci Rep 5: 8049 (2015)

[133] Ma J, Zhao Q, Meng Y. Magnetically controllable Casimir force based on a superparamagnetic metametamaterial. Phys Rev B 89: 075421 (2014)

[134] Hu Z D, Yan H, Qiu H Z, Zhang P, Liu Q. Friction and wear of magnetorheological fluid under magnetic field. Wear 278-279: 48-52 (2012)

[135] Chen W, Huang W, Wang X. Effects of magnetic arrayed films on lubrication transition properties of magnetic fluid. Tribol Int 72: 172-178 (2014)

[136] Wu Y, Wei Q, Cai M, Zhou F. Interfacial friction control. Adv Mater Interf 2(2): 1400392 (2015) 
[137] Lyashenko I A, Khomenko A V. Thermodynamic theory of two rough surfaces friction in the boundary lubrication mode. Tribol Lett 48(1): 63-75 (2012)

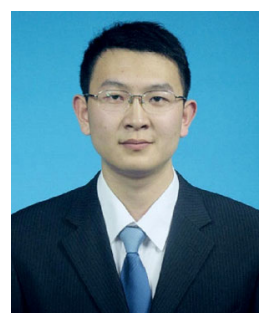

Jun ZHANG. He received his bachelor degree in mechanical engineering in 2011 from Tsinghua University, Beijng, China. After then, he was a Ph.D student in the State

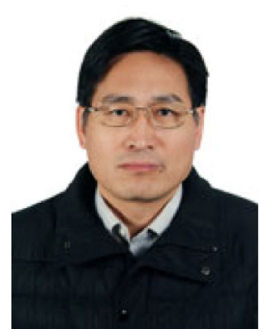

Yonggang MENG. He received his M.S. and Ph.D degrees in mechanical engineering from Kumamoto University, Japan, in 1986 and 1989 respectively. He joined the State Key Laboratory of Tribology at Tsinghua
[138] Wojciechowski Ł, Mathia T G. Conjecture and paradigm on limits of boundary lubrication. Tribol Int 82: 577-585 (2015)

Key Laboratory of Tribology at the same university. His research interests include adsorption of surfactant at solid/liquid interface, boundary lubrication and potential controlled friction.

University from 1990. His current position is a professor and the Director of the laboratory. His research areas cover the tribology of MEMS and hard disk drives, active control of friction and interfacial phenomena and nanomanufacturing. 